\title{
Intraoperative consultation for ovarian tumors
}

\author{
Insun Kim \\ Department of Pathology, Jinju Korea Hospital, Jinju, Korea
}

Received: April 23, 2019

Revised: May 30, 2019

Accepted: May 31, 2019

Corresponding author:

Insun Kim

Department of Pathology, Jinju

Korea Hospital, 2, Dongjin-ro, Jinju

52726, Korea

Tel: $+82-55-750-2570$

Fax: +82-55-757-4705

E-mail: iskim@korea.ac.kr
The primary function of intraoperative frozen consultation is to provide an as accurate and prompt diagnosis as possible during surgery and to guide the surgeon in further management. However, the evaluation of frozen section (FS) is sometimes difficult because of suboptimal tissue quality and frozen artifacts compared with routinely processed tissue section. The pathologist responsible for the FS diagnosis requires experience and good judgment. Ovarian tumors are a heterogeneous group of tumors including primary surface epithelial tumors, germ cell tumors and sex cord-stromal tumors, secondary tumors, and other groups of tumors of uncertain histogenesis or nonspecific stroma. Intraoperative FS is a very important and reliable tool that guides the surgical management of ovarian tumors. In this review, the diagnostic key points for the pathologist and the implication of the FS diagnosis on the operator's decisions are discussed.

Keywords: Frozen section; Intraoperative consultation; Ovarian tumors

\section{Introduction}

Intraoperative frozen consultation is one of the most important and difficult tasks of pathologists. Frozen section (FS) is performed during surgery, from which the pathologist provides a preliminary diagnosis, hence guiding the surgeon in further management. The three main purposes of FS are to establish the presence and nature of a lesion, to determine the adequacy of the surgical margins, and to establish whether the obtained tissue contains diagnostic material or whether additional sampling is needed [1]. The indications and limitations of FS vary from organ to organ. However, this procedure should not be used by surgeons to satisfy their curiosity, to recognize normal anatomic structures, or to communicate immediately with the patient's relatives. The pathologist responsible for the FS diagnosis requires experience, knowledge of clinical medicine and pathology, good judgment, and an awareness of the limitation of the FS method. To effectively obtain the result, the pathologist should review the patient's clinical history and ideally have a discussion with the surgeon before the operation.
When the fresh tissue is received, the pathologist should select the best area from the specimen according to the purpose of the FS. The tissue is quickly frozen using liquid nitrogen, sectioned with cryostat, stained with hematoxylin-eosin, and evaluated under the microscope by the pathologist. Special stains and immunostains as well as cytologic smears obtained through touch preparation can be added [2]. Compared with routine pathologic diagnosis of formalin-fixed paraffin-embedded tissue preparations, FS evaluation is limited by suboptimal tissue quality, frozen artifacts, time limitation, and lack of ancillary studies. It is also affected by the pathologist's experience, supportive persons, availability of a subspecialty pathologist, concurrent multiple FS specimens, and technical problems such as issues related to the instruments or the skill of the technician [3].

Gynecologic tumors are one of the most frequently encountered FS specimens in pathology laboratories. However, the evaluation of these lesions is often difficult because of the numerous disease entities and morphologic diversities, as well as their variants. Especially, ovarian tumors are a heterogeneous group of tumors including primary surface epithelial tumors, germ cell tumors and sex 
cord-stromal tumors, secondary tumors, and other groups of tumors of uncertain histogenesis or nonspecific stroma [4].

Helpful information for the FS diagnosis of an ovarian tumor includes the patient's age; relevant clinical and familial history; history of malignancy; serum markers such as alpha-fetoprotein, carcinoembryonic antigen, carcinoma antigen 125 , carbohydrate antigen 19.9, and human chorionic gonadotropin; hormone levels (estrogen, androgen); and imaging studies. A high concordance between FS diagnosis and final permanent section diagnosis has been reported, and the overall accuracy of FS diagnosis ranges between $80.7 \%$ and $97.1 \%$ for primary ovarian tumors $[5,6]$. However, the ultimate aim of FS in ovarian lesions is providing the surgeon with helpful information for the next step of the operation, rather than providing a specific pathologic diagnosis. If the tumor is benign, no further surgery is indicated. In borderline tumors, total abdominal hysterectomy and bilateral salpingo-oophorectomy (TAH-BSO) and staging procedure are indicated in postmenopausal women; however, unilateral salpingo-oophorectomy $(\mathrm{SO})$ and staging operation are indicated in young patients. In patients with ovarian carcinoma, TAH-BSO and debulking with staging procedure are recommended, whereas young patients with malignant sex cord-stromal tumors and germ cell tumors are suggested to undergo unilateral SO and staging procedure. Intraoperative FS is very important for guiding the surgical management of ovarian tumors.

In this review, the clinicopathologic, gross, and microscopic features of ovarian tumors are discussed, with special emphasis on the diagnostic key points and the implication of the FS diagnosis on the operator's decision during surgery. The materials and data collected from four university hospitals (Korea Anam, Inje Busan Paik, Gyeongsang, and Dankook Cheil) between 2016 and 2017 were reviewed, and the classification of ovarian tumors followed the 2014 World Health Organization classification of tumors of the female reproductive organs [4].

\section{General categories of ovarian tumors}

A total of 491 cases were submitted for frozen diagnosis of ovarian tumors. Excluding nonneoplastic and nonovarian lesions, 446 tumors were primary ovarian tumors $(95.5 \%)$ and 21 tumors were metastatic (4.5\%) on permanent diagnosis. Among the primary tumors, 372 were surface epithelial tumors (83.4\%), 43 were sex cord-stromal tumors $(9.6 \%)$, and the remaining 31 were germ cell tumors $(7.0 \%)$.

Surface epithelial tumors were classified into 166 mucinous tumors (44.6\%), 120 serous tumors (32.3\%), 43 endometrioid tumors (11.6\%), 16 clear cell tumors (4.3\%), 15 seromucinous tu- mors (4.0\%), and 2 Brenner tumors (0.5\%). The remaining 10 cases $(2.7 \%)$ comprised 4 mixed carcinomas, 4 malignant mixed Mullerian carcinomas, 1 undifferentiated carcinoma, and 1 endometrial stromal sarcoma. Sex cord-stromal tumors were classified into 30 fibroma-thecoma tumors (69.8\%) (19 fibromas, 6 cellular fibromas, 4 thecofibromas, and 1 thecoma), 11 granulosa cell tumors (GCTs) (25.6\%), and 2 Sertoli-Leydig cell tumors (SLCTs) (4.6\%). Germ cell tumors were classified into 23 mature cystic teratomas (74.2\%), 3 dysgerminomas (9.7\%), 2 immature teratomas $(6.5 \%), 1$ yolk sac tumor $(3.2 \%), 1$ choriocarcinoma (3.2\%), and 1 mixed tumor (3.2\%). The metastatic tumors comprised 17 adenocarcinomas, 2 malignant lymphomas, 1 squamous cell carcinoma, and 1 atypical carcinoid.

Thirty-six tumors reported as "benign" on FS were finally diagnosed as mucinous cystadenoma in 12 cases, endometriosis in 10 cases, serous cystadenoma in 9 cases, teratoma in 3 cases, mucinous borderline tumor (MBT) in 1 case, and seromucinous tumor in 1 case. Twenty-eight tumors reported as "adenocarcinoma" or "carcinoma" were finally diagnosed as serous carcinoma in 15 cases, endometrioid carcinoma (EC) in 4 cases, clear cell carcinoma in 2 cases, mucinous carcinoma in 2 cases, carcinosarcoma in 2 cases, and metastatic tumor in 2 cases.

\section{Surface epithelial tumors}

Tumors of surface epithelial origin are the most commonly encountered group in ovarian FS. Intraoperative FS evaluation is very important for determining the extent of surgery. In malignant tumors, staging laparotomy including total abdominal hysterectomy with bilateral SO, lymphadenectomy, peritoneal sampling, and omentectomy should be done, whereas limited cystectomy or unilateral SO is done for benign tumors, especially in young patients who want to preserve their fertility. Misinterpretation of FS may lead to unnecessary extensive surgery or a risk for a second operation. The distinction between borderline tumors and invasive cancers is sometimes difficult. A conservative approach may be appropriate, especially in young patients. The findings of careful gross examination, bilaterality, clinical history of a previous malignancy, and the patient's age and reproductive status are important in intraoperative consultation. In addition, the differential diagnosis of surface epithelial tumors may include other primary ovarian neoplasms.

\section{Mucinous tumors}

Mucinous tumors are classified into benign cystadenoma/cystadenofibroma/adenofibroma, MBT/atypical proliferative mucinous tumor (APMT), and mucinous carcinoma. Mucinous tu- 
mors are characterized by cyst and glands lined by epithelial cells containing intracytoplasmic mucin. They tend to be the largest of all ovarian tumors, with many of them being $15-30 \mathrm{~cm}$ in diameter and are typically unilateral. Approximately $75 \%$ are benign, $10 \%$ are borderline, and $15 \%$ are carcinomas. In the review of 166 frozen diagnosis of mucinous tumors, $67.5 \%$ were benign, $22.3 \%$ were borderline, and $10.2 \%$ were malignant.

\section{1) Mucinous cystadenoma/cystadenofibroma/adenofibroma}

Benign mucinous tumors are cystic, unilocular or multilocular, and contain viscous, mucoid material. If the tumor is partly or entirely solid, cystadenofibroma or adenofibroma is considered. The important point in intraoperative consultation is the distinction from other benign cysts, if possible, because a metastatic mucinous tumor from the appendix may sometimes mimic a primary benign mucinous cystic tumor. The surgeon may explore the appendix and perform appendectomy to exclude the possibility of an appendix origin. An additional FS or additional sampling for permanent section may be indicated in mucinous cystadenoma with focal epithelial proliferation and atypia.

Among 93 tumors diagnosed as mucinous cystadenoma on FS, 11 tumors were diagnosed as borderline, 8 as focally proliferative, and 2 as teratomas ( 1 mature and 1 immature) on permanent diagnosis. Twenty-two tumors diagnosed as cystadenoma with focal proliferation were diagnosed as borderline in 6 cases, cystadenoma in 4 cases, and seromucinous tumor in 1 case. Upgrading to a borderline tumor was seen in cases of cystadenoma (Fig. 1) and cystadenoma with focal proliferation. The distinc- tion from a seromucinous tumor is not crucial on FS. Because mucinous ovarian tumors are commonly heterogeneous with a morphologic spectrum from benign to borderline to malignant areas, adequate sampling from the most solid area is very important for a correct diagnosis.

2) Mucinous borderline tumors/atypical proliferative mucinous tumors

Multilocular cystic tumors contain viscous mucoid material. A solid component is uncommon; however, if it is present, carcinoma or mural nodule should be considered. Because the tumor is heterogeneous in morphology, multiple areas should be sampled for FS. Epithelial proliferation is seen in $>10 \%$ of tumors, but no stromal invasion is noted.

MBT/APMT with intraepithelial carcinoma represents mucinous tumors showing areas of stratification to 4 or more layers or a cribriform pattern with severe nuclear atypia, but no stromal invasion. MBT/APMT with microinvasion indicates MBT with stromal invasion of $<5 \mathrm{~mm}$.

Four tumors among $18 \mathrm{MBTs} / \mathrm{APMTs}$ and 2 of $4 \mathrm{MBTs} / \mathrm{AP}$ MTs with microinvasion were diagnosed as mucinous carcinomas on permanent section (Fig. 2). Five of 6 tumors reported as MBTs/APMTs rather than mucinous carcinomas were finally diagnosed as mucinous carcinomas.

A definite diagnosis of intraepithelial carcinoma or microinvasion, and the distinction from seromucinous borderline tumor (SMBT) are not necessary in FS. The frozen diagnosis of MBT/ APMT is adequate. If the distinction from a mucinous carcinoma
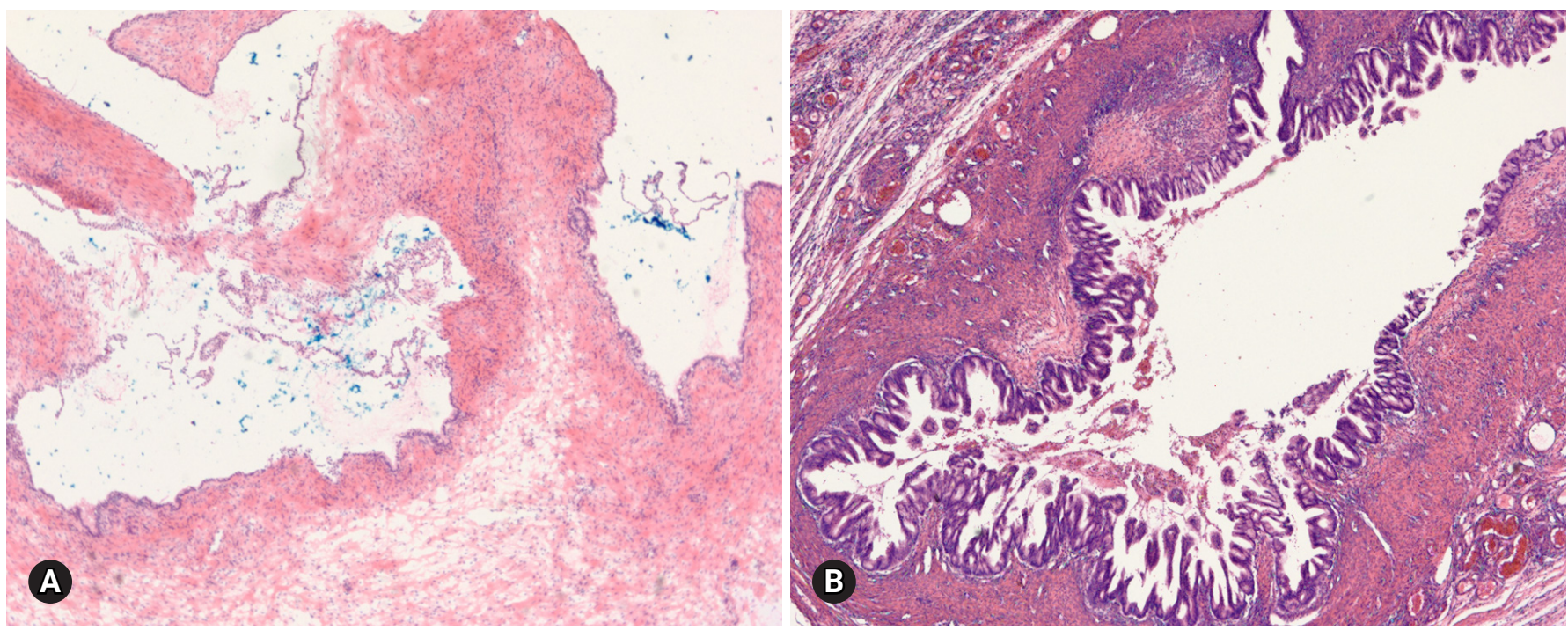

Fig. 1. On frozen section, the multilocular cyst is lined by single layer of tall columnar cells (A), but the epithelial cells are stratified and show papillary growth indicating mucinous borderline tumor on permanent section (B). 

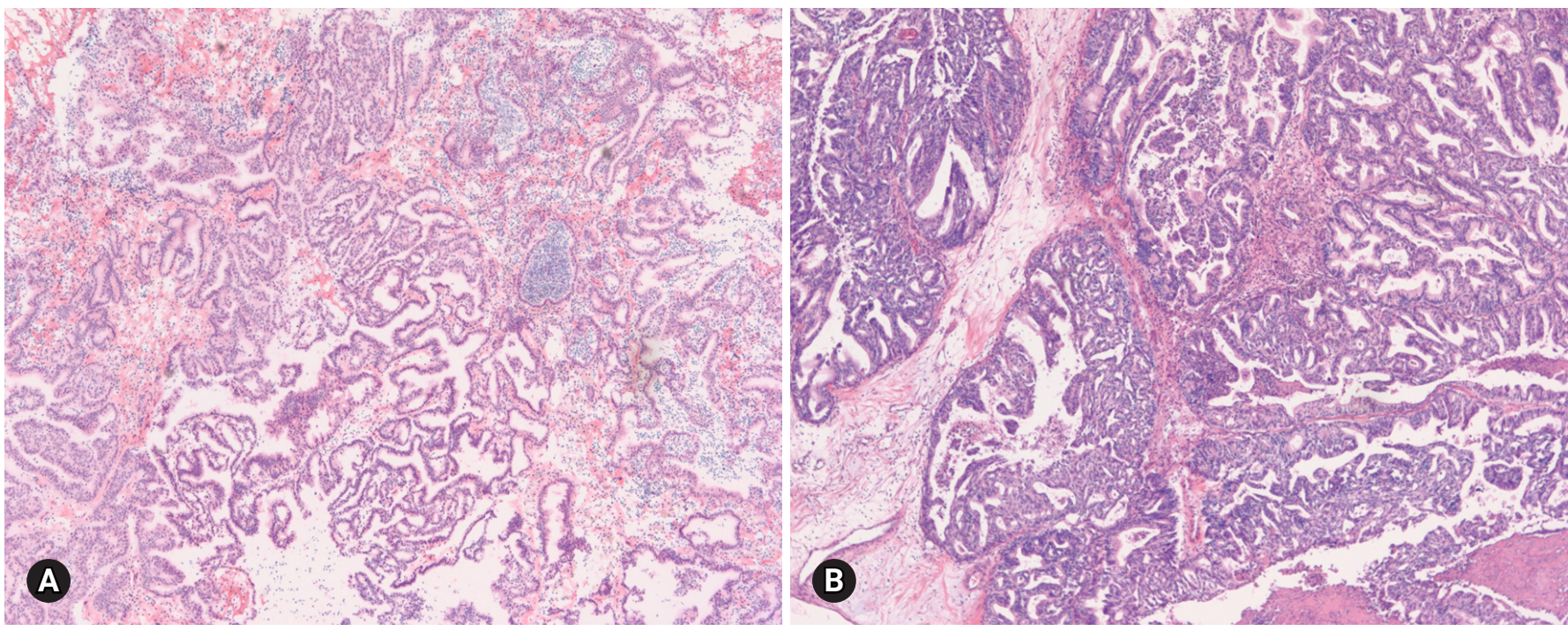

Fig. 2. On frozen section, the tumor was diagnosed as mucinous borderline tumor (A), but permanent histologic section shows expansile type of mucinous carcinoma (B).
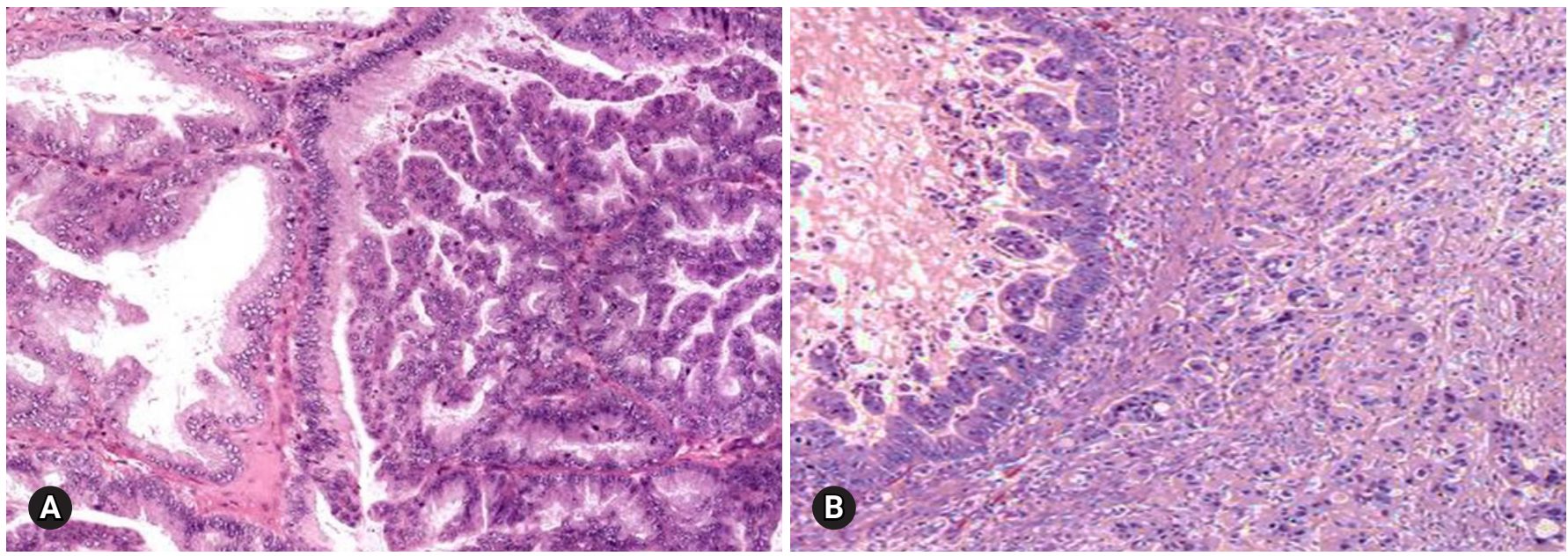

Fig. 3. Two patterns of invasion in mucinous carcinoma: expansile (A) and infiltrative (B).

is difficult, a frozen diagnosis of "at least MBT" can be made.

Because of the excellent prognosis, even with intraepithelial carcinoma or microinvasion, fertility-sparing surgery is adequate for young patients with MBT/APMT [7].

\section{3) Mucinous carcinomas}

Mucinous carcinoma is a relatively rare histologic subtype and presents with a large, unilateral mass $(>10 \mathrm{~cm})$. The tumor is complex and multicystic with solid areas. Necrosis and hemorrhage may be present. Two patterns of stromal invasion $(>5$ $\mathrm{mm})$, expansile (confluent) and destructive (infiltrative), should be identified (Fig. 3). The tumor may have areas resembling a benign cystadenoma or a borderline tumor (Fig. 4). Multiple ad- equate samplings from solid areas and areas near necrosis are key for the intraoperative FS diagnosis of MBT/APMT and mucinous carcinoma, because of the heterogeneous morphologic continuum from benign to borderline to malignant areas.

Among 7 tumors that were diagnosed as mucinous carcinoma on FS, 1 tumor was a clear cell carcinoma and 1 tumor was a metastatic carcinoma. The distinction from metastatic carcinoma is very important, because surgical staging should be done for a primary ovarian tumor but not for a metastatic carcinoma. Bilaterality, small tumor size $(<10 \mathrm{~cm})$, and the patient's clinical history of a prior malignancy indicate a metastatic carcinoma rather than a primary tumor [8]. The presence of a teratomatous component suggests a primary ovarian tumor. 

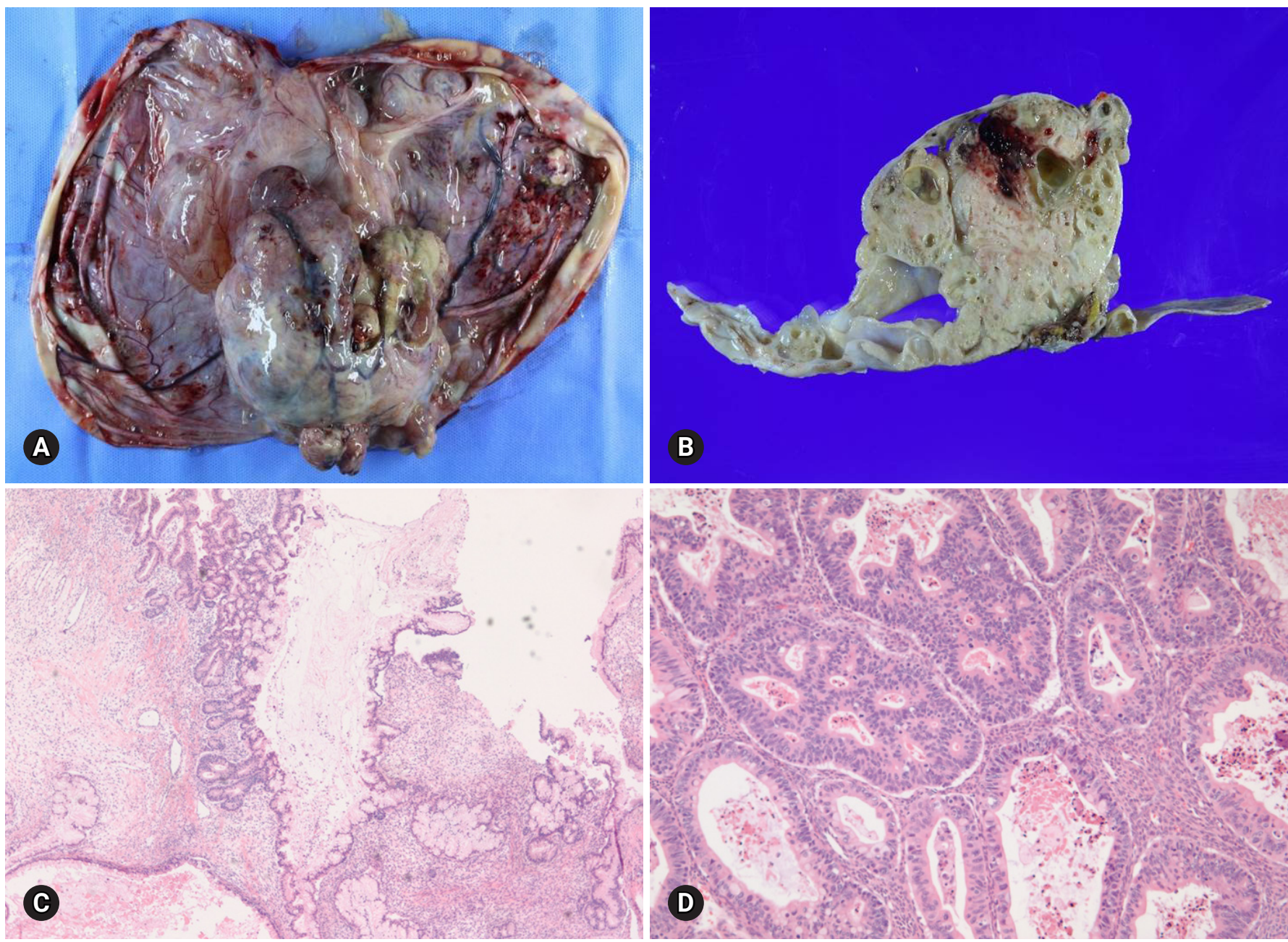

Fig. 4. Goss finding of mucinous carcinoma is cystic, with polypoid mass (A). On section, the solid mass is sponge-like with hemorrhage and necrosis (B). The cystic area is mucinous borderline tumor (C), but solid portion is mucinous carcinoma (D).

4) Mucinous tumors with mural nodules

Reactive sarcoma-like mural nodules and malignant mural nodules are rarely associated with benign, borderline, or malignant mucinous tumors. A reactive mural nodule typically occurs in younger patients and does not alter the prognosis, whereas a malignant mural nodule characterized by anaplastic carcinoma or sarcoma is usually seen in older patients and has a poor prognosis (Fig. 5) $[9,10]$. High-grade carcinoma/sarcoma associated with a mucinous tumor may be enough for frozen diagnosis, and the final diagnosis can be deferred for permanent sections.

\section{Serous tumors}

Serous tumors are classified as benign cystadenoma/cystadenofibroma/adenofibroma, serous borderline tumor (SBT)/ atypical proliferative serous tumor (APST), SBT/APST micropapillary variant/noninvasive low-grade serous carcinoma (LGSC),

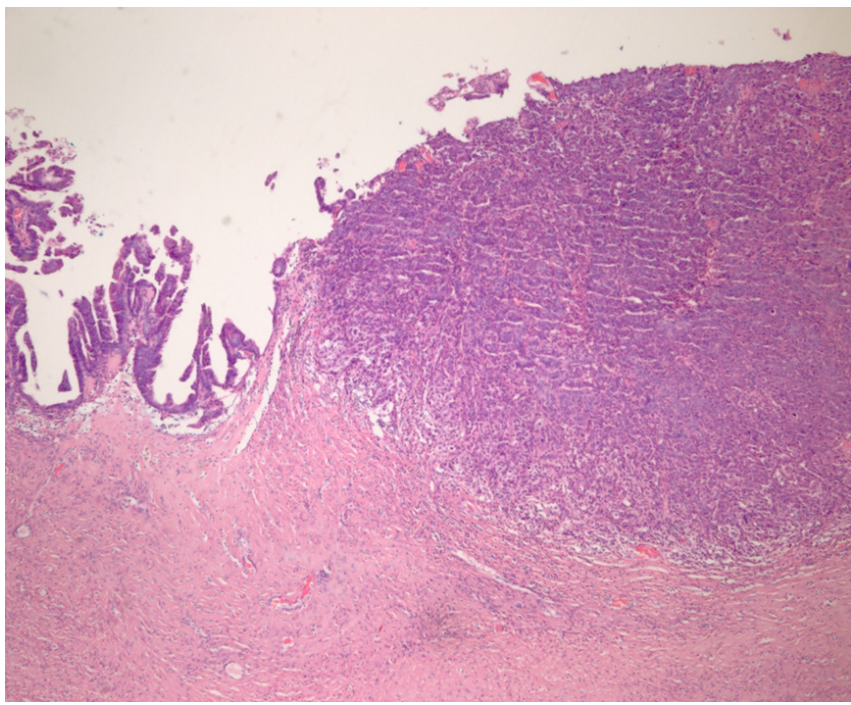

Fig. 5. Mucinous borderline tumor shows mural nodule composed of anaplastic carcinoma. 
invasive LGSC, and high-grade serous carcinoma (HGSC). Approximately $70 \%$ of serous tumors are benign, $10 \%$ are borderline, and 20\% are carcinomas. However, among 120 serous tumors on FS, borderline and malignant tumors accounted for $22.5 \%$ and $43.3 \%$, respectively, and benign tumors comprised only $24.2 \%$.

\section{1) Serous cystadenoma/cystadenofibroma/adenofibroma}

Benign serous tumors usually occur in adults, and bilaterality is observed in about $20 \%$. The tumor is a unilocular or an oligolocular cyst containing clear watery fluid. In cystadenofibroma, the tumor is cystic and solid and shows firm papillary projection, whereas the tumor is solid and firm with small spaces in adenofibroma. Epithelial proliferation involves $<10 \%$ of the epithelial lining [11]. If the frozen diagnosis is cystadenoma with focal epithelial proliferation, additional frozen sampling is recommended; however, no staging is required. The distinction from benign, nonneoplastic cyst is not important, but mucinous tumor should be ruled out because appendectomy may be done to exclude metastasis from a primary appendix tumor.

Among 22 benign serous tumors on frozen diagnosis, 1 tumor was borderline and 2 tumors were mucinous tumors. Among 4 benign versus borderline tumors, 1 was benign and the other was SMBT.

The distinction from a mucinous tumor should be based on the histologic cell type, not on the cystic content. The papillary projection in benign tumors is typically firm and small in number, whereas papillary growth in SBT/APST is soft, friable, and large in number.
2) Serous borderline tumor/atypical proliferative serous tumor SBT/APST is bilateral in up to 55\% of cases, and typically cystic. Abundant friable papillary projections are present on the inner lining of the cyst and/or a cauliflower-like mass may be present on the ovarian surface without a cystic component (serous surface papillary borderline tumor). Microscopically, epithelial proliferation is seen in at least $10 \%$ of tumors (Fig. 6). A micropapillary or cribriform pattern may also be seen $(<5 \mathrm{~mm})$. Psammomatous calcification, which may be observed in benign and malignant serous tumors, is found in approximately one-fourth of the cases. Microinvasion ( $<5 \mathrm{~mm}$ in greatest dimension) may be present. The distinction from serous cystadenoma with or without focal epithelial proliferation $(<10 \%)$ is sometimes difficult on FS. A conservative approach is recommended, especially in young patients. If the stromal invasion is $>5 \mathrm{~mm}$, it should be classified as invasive LGSC. If the micropapillary pattern is $>5$ $\mathrm{mm}$, it should be classified as noninvasive LGSC, micropapillary variant $[11,12]$. However, the distinction from noninvasive or invasive low-grade carcinoma is not crucial at the time of FS. "At least SBT" or "SBT with micropapillary features" is an appropriate FS diagnosis. HGSC requiring complete surgical staging should be ruled out on FS.

SBT/APST is associated with peritoneal implants in about $30-40 \%$ of the cases. The implants are classified into the noninvasive and invasive types, and the noninvasive type is subdivided into the epithelial and desmoplastic subtypes [13]. Invasive implants behave like LGSC [4]. The distinction between noninvasive implants of SBT/APST and LGSC may not be crucial at the time of FS, especially in older patients. Additional sampling from
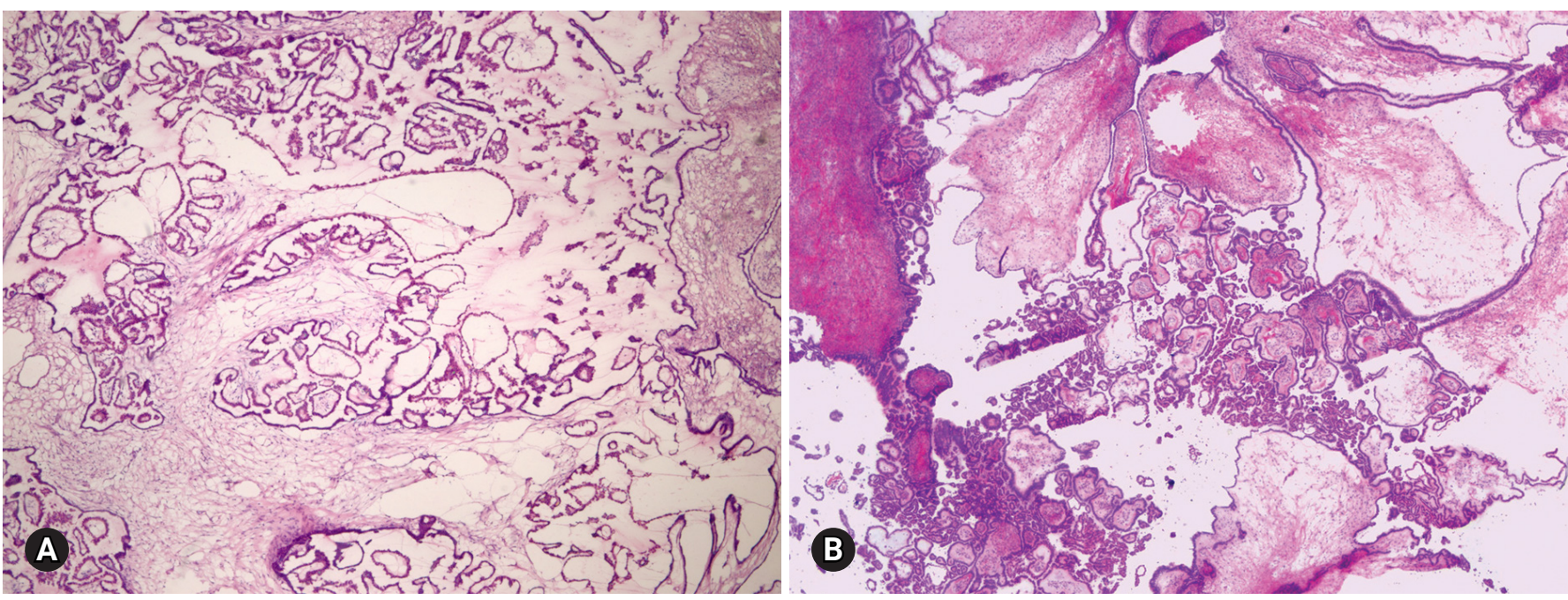

Fig. 6. Serous borderline tumor on frozen section (A) and on permanent section (B). Epithelial proliferation is characterized by hierarchical branching papillae with budding and tufting, and stratification of the cells. 
the peritoneum or ovary may be helpful for a specific diagnosis.

Among 17 SBTs/APSTs on FS, 1 tumor was SMBT. Among 6 borderline versus malignant tumors, 3 tumors were malignant and 1 was SMBT. The distinction from SMBT has no impact on the surgical management at the time of FS.

\section{3) Noninvasive low-grade serous carcinoma}

The clinical and gross findings of noninvasive LGSC (SBT/ APST micropapillary variant) are similar to those of SBT/APST.

Microscopically, it is characterized by a nonhierarchical branching micropapillary or cribriform architecture in at least 1 confluent area measuring $5 \mathrm{~mm}$ [6]. Micropapillae are defined as at least 5 times taller than wide, with scant or no fibrovascular cores. The distinction from SBT/APST may not be crucial at the time of FS. "At least SBT" or "SBT with micropapillary features" may be enough for the frozen diagnosis, and fertility-sparing surgery may be done in young patients.

4) Low-grade serous carcinoma

LGSC is relatively rare and commonly advanced at the time of presentation. It is often bilateral and solid or partly cystic, with papillary growth. Microscopically, it is characterized by relatively uniform nuclei with mild to moderate atypia, and mitotic figures $<12 / 10$ high-power fields (HPFs) [14]. Psammoma bodies may be abundant. Destructive stromal invasion $(>5 \mathrm{~mm}$ ) is present. The distinction from SBT/APST may not be crucial at the time of FS. Additional biopsy from extraovarian lesions or implants may be helpful in identifying destructive stromal invasion. The value of fertility-sparing surgery is not well documented in this group, and the prognosis is dependent on the disease stage.

Pelvic or para-aortic lymph nodes are not infrequently involved by the papillary or glandular structures, similar to primary ovarian SBT/APST or LGSC. The distinction between primary nodal proliferation and metastasis from the ovary may be difficult. However, both findings do not change the prognosis and do not influence the treatment. Occasionally, extensive lymph node involvement in patients with invasive peritoneal implants is associated with a poor prognosis.

The distinction from HGSC should be done on FS. Highgrade carcinoma characterized by marked nuclear atypia, numerous mitotic figures, and necrosis is different from low-grade carcinoma [14]. Unfortunately, most of the cases were not adequately diagnosed as LGSC or HGSC in this review.

\section{5) High-grade serous carcinoma}

HGSC is the most common type of ovarian carcinoma. It occurs in older age groups, presents at an advanced stage, and is bilateral in the majority of the cases. Grossly, the tumor is solid or solid and cystic with frequent involvement of the ovarian surface. Hemorrhage and necrosis are common. Histologically, the tumor is characterized by an admixture of solid, complex glandular growth with slit-like spaces, cribriform, and papillary patterns (Fig. 7). Marked nuclear atypia with prominent nucleoli, pleomorphism, and numerous mitotic figures ( > 12/10 HPFs) with atypical forms are present [14]. Stromal invasion is present. A tumor with an intracystic papillary pattern showing marked nuclear atypia and numerous mitotic figures, but without stromal invasion, is also classified as HGSC. The important issue in FS is the recognition of high-grade epithelial malignancy. The distinction from LGSC is the presence of marked nuclear atypia and numerous mitotic figures ( $>12 / 10 \mathrm{HPFs}$ ). The differential diagnosis from other high-grade carcinomas of Mullerian origin, endometrioid, clear cell carcinoma, and malignant Brenner tumor is not important. "High-grade carcinoma, consistent with a Mullerian origin" may be adequate in difficult cases. HGSC may arise from the fallopian tube or peritoneum and metastasizes from endometrial serous carcinoma. The distinction from these primary sites is not crucial on FS.

\section{Endometrioid tumors}

Endometrioid tumors include benign endometriotic cyst and cystadenoma/adenofibroma, endometrioid borderline tumor (EBT)/atypical proliferative tumor (APET), and malignant carcinoma. In the review of 43 frozen diagnoses, endometriotic cysts accounted for $70 \%$, malignant tumors accounted for $25 \%$, and borderline tumors accounted for $5.0 \%$.

\section{1) Endometriotic cyst/cystadenoma/adenofibroma}

Endometriotic cyst is a cystic form of endometriosis. Histologically, the cyst is lined by endometrial epithelium and underlying endometrial stroma, but cystadenoma/adenofibroma is lacking the endometrial stroma. The distinction from other benign lesions is not crucial on frozen diagnosis. Endometriosis with cytologic atypia or atypical hyperplasia should be managed conservatively. However, adequate sampling is important because it may be associated with borderline and malignant endometrioid, clear cell, and seromucinous tumors.

In the review of 20 benign endometriotic lesions, 1 case was associated with clear cell carcinoma and the other was a mucinous cystadenoma. Sampling from a polypoid or solid lesion of an endometriotic cyst is considered important.

2) Endometrioid borderline tumor/atypical proliferative tumor $\mathrm{EBT} / \mathrm{APET}$ is an uncommon tumor that is predominantly solid 

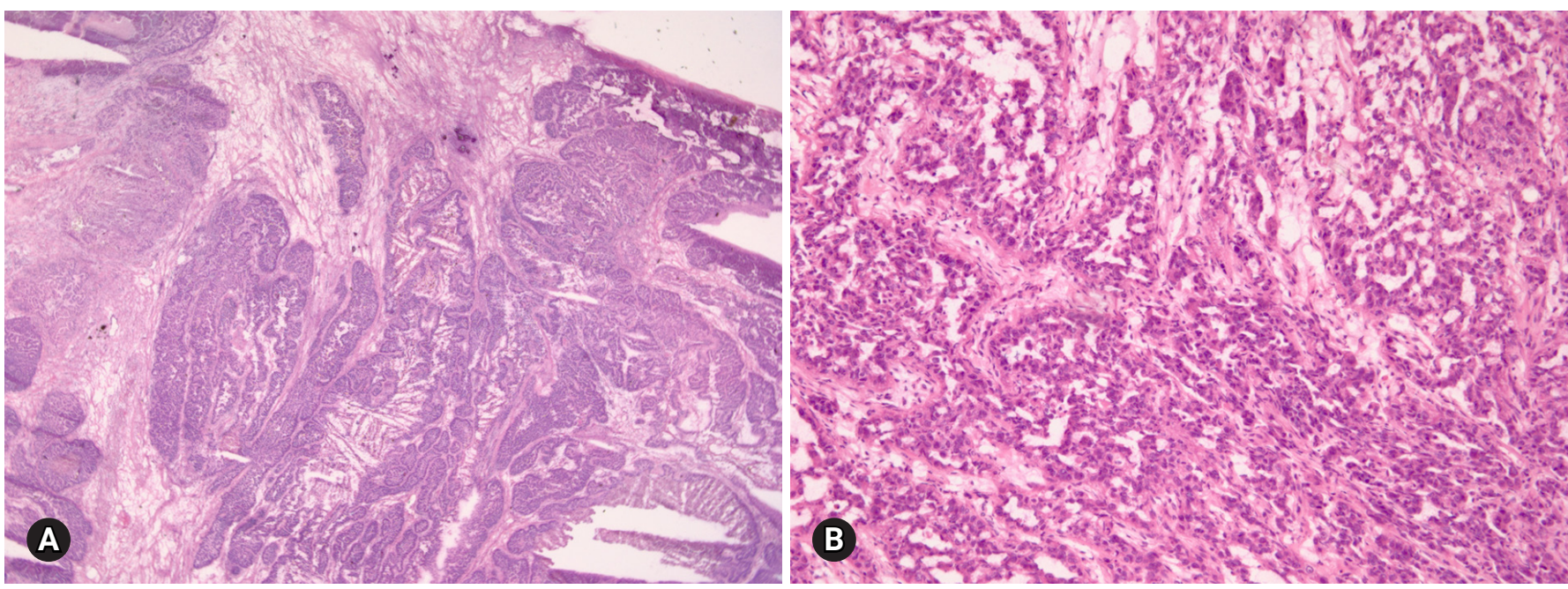

Fig. 7. High grade serous carcinoma shows solid and complex glandular growth patterns and psammomatous calcification on frozen section (A), and slit-like spaces of tumor cells with marked nuclear atypia (B).

but shows a focally cystic or sponge-like cut surface. Histologically, the tumor shows complex, crowded glandular structures in a fibromatous or intracystic pattern. Squamous metaplasia is common. Mild to moderate nuclear atypia, but without stromal invasion, is present. EBT/APET with intraepithelial carcinoma is defined as marked cytologic atypia but without stromal invasion, whereas EBT/APET with microinvasion is defined as confluent glandular growth or stromal invasion of $<5 \mathrm{~mm}$ dimension.

Complete surgical staging is the standard surgical management for EBT/APET, but fertility-sparing surgery may be an option for young patients because of their excellent prognosis. The presence of intraepithelial carcinoma or microinvasion does not change the surgical management, but adequate sampling is recommended to confirm unequivocal invasion $[15,16]$. EC may arise in the background of EBT/APET or endometriosis. Six endometrioid tumors were interpreted as borderline versus malignant on FS, and 3 of them were finally diagnosed as EC.

\section{3) Endometrioid carcinoma}

ECs occur in older age groups, and bilaterality is observed in up to $17 \%$ of the cases [4]. The tumor is a solid or blood-filled cyst with intraluminal soft mass or polypoid nodule. Histologically, the tumor resembles endometrial EC. The stromal invasion should be $>5 \mathrm{~mm}$. Squamous differentiation is often present, in up to $30-50 \%$ of the cases (Fig. 8) [17]. Adequate sampling from solid and necrotic areas is important to rule out EC in the case of EBT/APET.

The distinction from other primary ovarian serous, clear cell, and seromucinous carcinomas is not crucial; however, mucinous differentiation should be ruled out because of the possibility of a metastatic carcinoma. Metastatic carcinoma from a primary colorectal and endocervical tumor may mimic EC. Squamous differentiation and an association with EBT/APET or endometriosis in the background are the most helpful features indicating a primary ovarian origin. The "garland" pattern with intraluminal dirty necrosis is the feature indicative of metastasis. Information on the patient's history of prior malignancies or concurrent lesions should be obtained from the surgeon. Simultaneous ovarian and endometrial ECs are present in 15-20\% of the cases [18]. Whether the ovarian tumor is a primary tumor or a metastasis from the endometrium is not important on frozen diagnosis. Among 9 ECs, only 1 case was correctly diagnosed on FS and the others were serous carcinoma ( 5 cases), seromucinous carcinoma ( 1 case), mixed MC and EC ( 1 case), and metastatic carcinoma (1 case).

\section{Clear cell tumors}

Benign tumors are exceptionally rare, and borderline tumors account for $<1 \%$. Most tumors are clear cell carcinomas. Clear cell tumors arise as a solid adenofibromatous or a cystic endometriotic type $[19,20]$. In the solid type, the cut surface is sponge-like with numerous small cysts (Fig. 9A). A benign tumor is microscopically characterized by benign-appearing glandular epithelium, whereas a borderline tumor shows atypical or malignant epithelium without invasion or microinvasion. A frankly invasive component ( $>5 \mathrm{~mm}$ in size) is present in a malignant tumor. A cystic tumor is unilocular or multilocular, with protruding polypoid masses in the lumens (Fig. 9B). The lining of the cyst rep- 

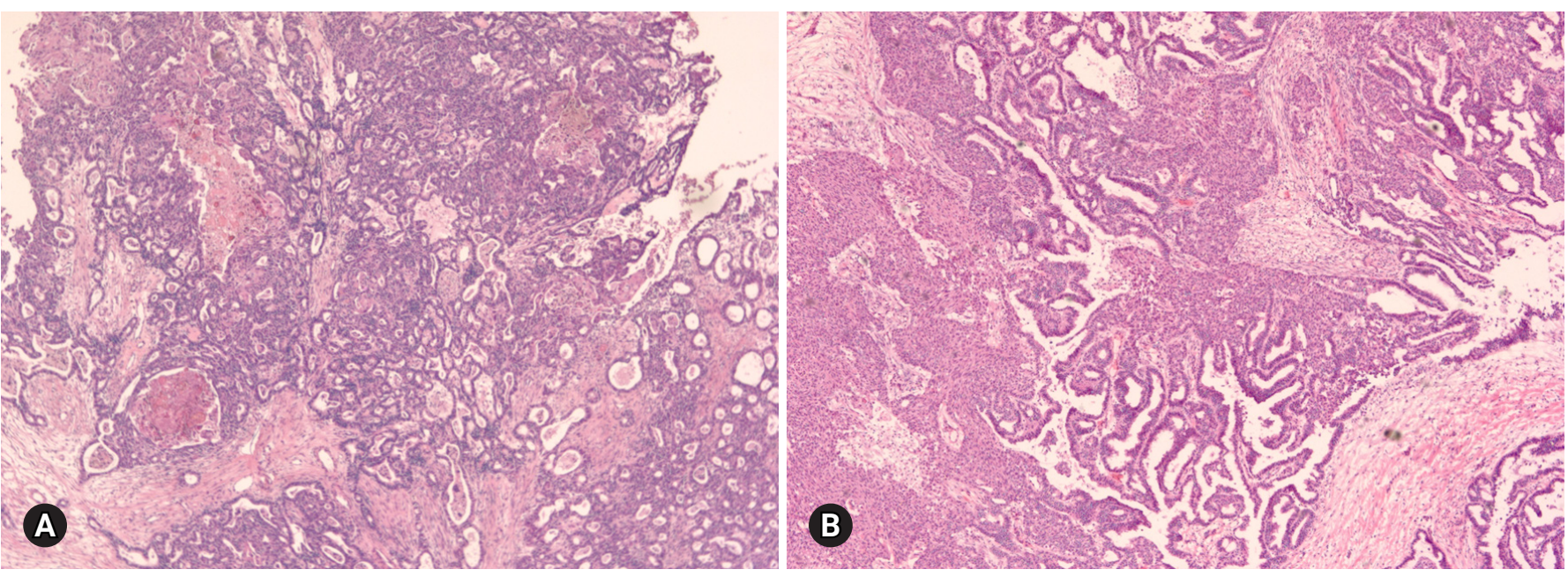

Fig. 8. Endometrioid adenocarcinoma shows confluent, back-to back glandular proliferation with loss of intervening stroma (A), and squamous component (B).
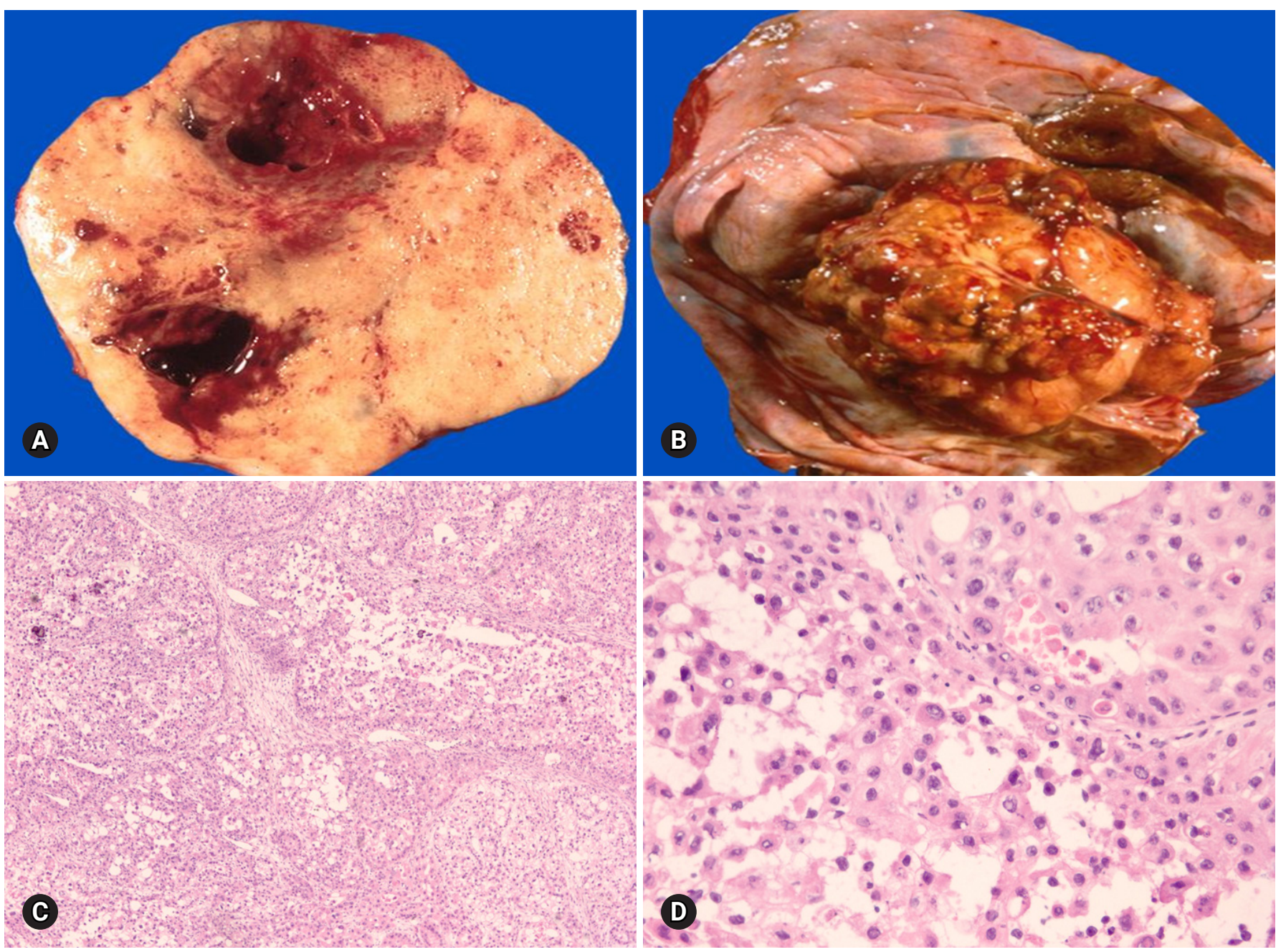

Fig. 9. Clear cell carcinoma may have a solid cut surface (A) or may show a predominantly cystic appearance with intraluminal solid growth (B). On frozen section, the tumor has solid and papillary growth patterns and psammomatous calcification (C). The tumor cells are large and pleomorphic. Hyaline bodies are found (D). 
resents benign or atypical endometriosis. The tumors are characterized by various cell types and histologic patterns (Fig. 9C). The most diagnostic features include dense hyalinized cores of papillae and hyaline bodies, which are present in $25 \%$ of the cases (Fig. 9D). Because a benign or borderline clear cell tumor is exceedingly rare, multiple additional sections should be taken to rule out carcinoma. Marked nuclear atypia is helpful in the differentiation of intracystic papillary clear cell carcinoma from SBT/ APST. The distinction from primary ovarian HGSC and EC as well as the distinction between metastatic clear cell carcinoma and Mullerian clear cell carcinoma are not crucial on FS. Yolk sac tumor and dysgerminoma should be ruled out, because fertility-sparing surgery is an option for young patients. Germ cell tumors typically occur in younger age groups $\left(2^{\text {nd }}\right.$ and $3^{\text {rd }}$ decades $)$ than clear cell carcinoma. All 11 clear cell carcinomas diagnosed on FS were correct; however, 3 tumors were diagnosed as adenocarcinoma and 1 as mucinous carcinoma. The other 1 case arising in association with endometriosis was misdiagnosed as endometriosis.

\section{Seromucinous tumors}

Seromucinous tumor is a mixed epithelial neoplasm with 2 or more Mullerian cell types, all accounting for at least $10 \%$ of the epithelium. The tumors are unilocular or paucilocular with papillary excrescences (Fig. 10A). Seromucinous tumors are associated with endometriosis in $30 \%$ of the cases (Fig. 10B) [4]. The most predominant epithelial cell types are serous and endocervical-type mucinous epithelium (Fig. 10C). The tumors are classified into benign seromucinous cystadenoma/ adenofibroma, borderline seromucinous tumor/atypical proliferative seromuci-
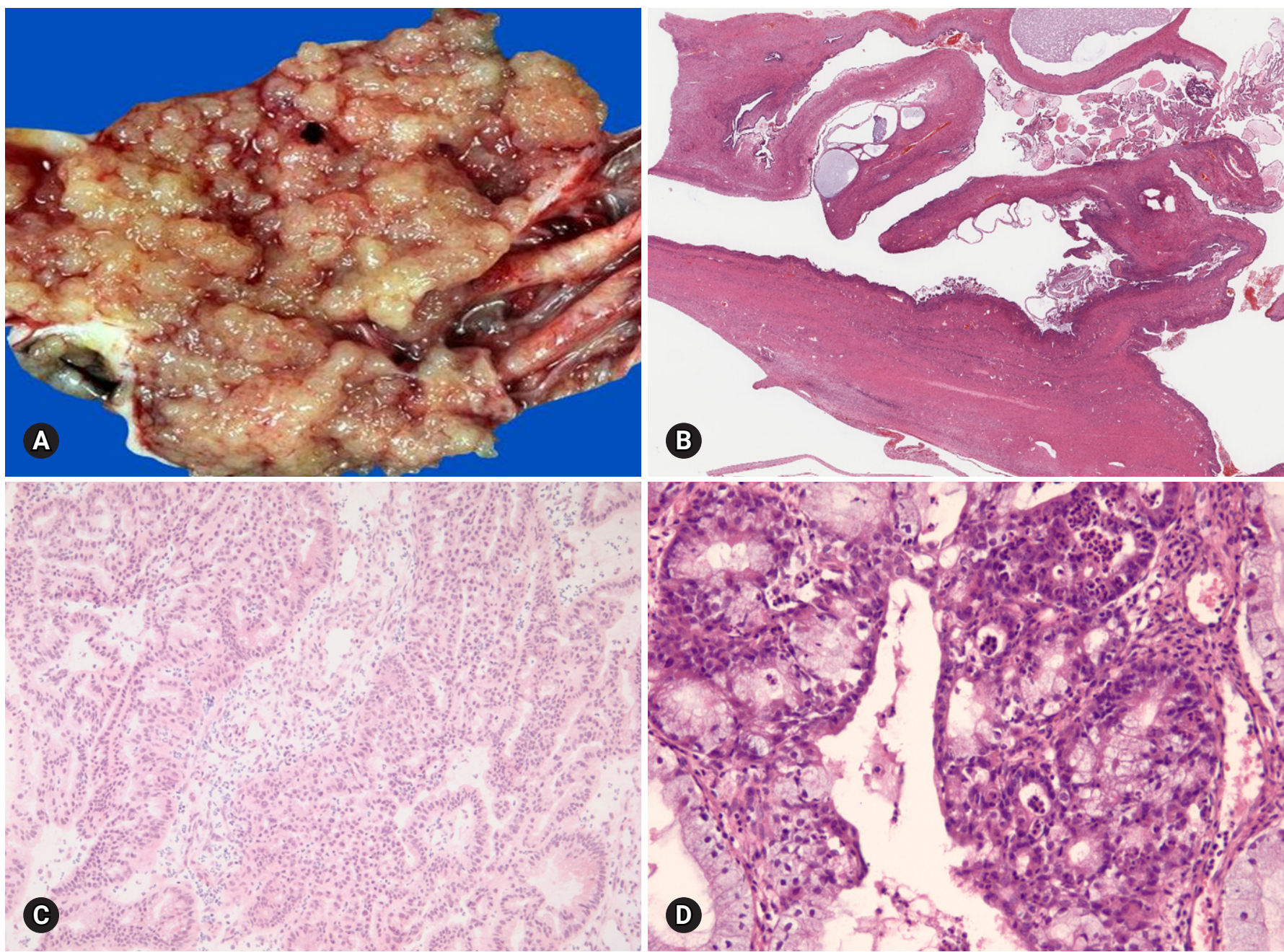

Fig. 10. Seromucinous borderline tumor is grossly cystic and shows intraluminal papillary growth (A). Histologically papillary growth is similar to serous borderline tumor (B). Frozen (C) and permanent (D) sections show complex glands composed of serous and mucinsecreting cells infiltrated with neutrophils. 
nous tumor, and malignant seromucinous carcinoma. Benign and malignant tumors are rather uncommon, and most tumors belong to the borderline category. These tumors are architecturally similar to SBT/APST, but larger papillae have edematous stroma containing neutrophils (Fig. 10D). Microinvasion, intraepithelial carcinoma, and a micropapillary pattern may occur.

On FS, the distinction from SBT/APST may be difficult; however, it is not important at the time of FS.

Seromucinous tumors were frequently diagnosed as serous tumors, but also as mucinous or endometrioid tumors in this review.

\section{Sex cord-stromal tumors}

Sex cord-stromal tumors account for approximately 5-10\% of all ovarian tumors and are commonly associated with estrogenic or androgenic manifestations. The tumors are classified into pure stromal tumors, pure sex cord tumors, and mixed sex cord-stromal tumors, and present a variety of histologic types [6]. Because the biologic behavior of sex cord-stromal tumors differs according to the histologic type, recognition of the specific entity may guide the intraoperative management.

\section{Fibroma-thecoma}

Fibroma is the most common type of stromal tumor. The tumor is solid, firm, white or tan, or hemorrhagic because of torsion (Fig. 11A). Microscopically, the tumor is composed of intersecting bundles and fascicles of spindle cells with hyalinization, collagen bands, or plaque (Fig. 11B). Cellular fibroma accounts for about $10 \%$ and is densely cellular with scant collagen. The number of mitosis is $\leq 3 / 10 \mathrm{HPFs}$, and nuclear atypia is absent or mild. A cellular tumor with mitotic activity of $\geq 4 / 10 \mathrm{HPF}$ and no more than mild nuclear atypia is defined as mitotically active cellular fibroma, whereas a tumor with at least moderate nuclear atypia and increased mitotic activity of $\geq 4 / 10 \mathrm{HPFs}$, often with atypical form, is termed as fibrosarcoma [21].

Thecomas are relatively uncommon tumors that typically occur in postmenopausal women with symptoms of estrogen production. The tumors are unilateral, and the cut surface is solid and yellow. The tumor is microscopically composed of sheets of oval or round cells with moderate to abundant pale eosinophilic cytoplasm. The tumor is designated as a fibrothecoma when an area resembling a fibroma is present.

On FS, the distinction of fibroma from thecoma is not important. However, the distinction from metastatic carcinoma with reactive stromal proliferation (Krukenberg tumor) is important. Bilaterality should raise a suspicion for metastasis, and careful microscopic examination to search for scattered tumor cells with a signet ring or breast lobular carcinoma should be done. Thorough sampling is recommended when minor sex cord elements are present, to rule out GCT or SLCT. Luteinized cells in thecomas may suggest the possibility of Leydig cell tumor or SLCT. Distinction from Leydig cell tumor is not crucial, but identification of a Sertoli cell component is important because surgical staging is required for SLCT [22].

Among 30 tumors, 1 fibrothecoma was suggested to be a metastatic poorly differentiated carcinoma and 3 cellular fibromas were diagnosed on permanent section.

\section{Granulosa cell tumors}

GCT is a low-grade malignant tumor composed of granulosa
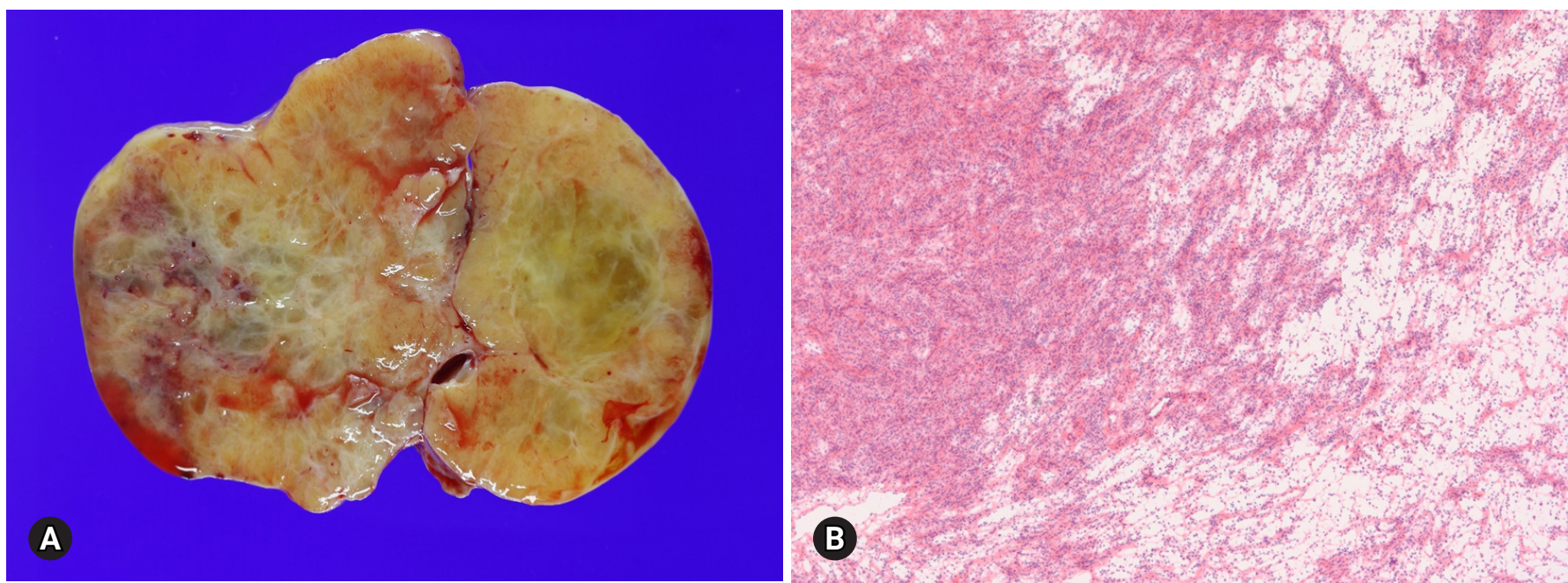

Fig. 11. Cut surface of fibroma is solid, firm, tan white and yellow (A). Histologically, the tumor is hypercellular and hypocellular with interlacing fascicles of spindle tumor cells (B). 
cells often with a variable number of fibroblasts and theca cells. The tumors comprise of two different clinicopathologic subtypes: adult and juvenile. The adult type more often occurs in postmenopausal patients, whereas the juvenile type mainly occurs in children and younger women. The patients may present abnormal vaginal bleeding associated with estrogenic manifestations. Patients with adult GCT have concurrent endometrial hyperplasia $(25 \%)$ or adenocarcinoma (5\%), and younger patients may present isosexual precocity [23]. The tumors are commonly unilateral, typically solid and cystic, and tan-yellow or white. Microscopically, the adult form is characterized by a mixture of various morphologic patterns, and the tumor cells are uniform with scanty cytoplasm and pale grooved nuclei (Figs. 12A, 12B). The juvenile form typically shows macrofollicles filled with basophilic secretions, as well as cells with moderate to abundant cytoplasm and darker nuclei usually without grooves [24].

The surgical treatment of GCT in postmenopausal patients in- cludes total hysterectomy and bilateral $\mathrm{SO}$ as part of the complete surgical staging procedure, whereas fertility-sparing surgery including unilateral $\mathrm{SO}$ and staging procedure may be done in younger patients with unilateral tumor without surface involvement $[25,26]$.

On FS, the distinction between the adult and juvenile forms is not crucial. The distinction of the adult type from benign sex cord-stromal tumors and metastatic carcinoma is the most important, because these lesions do not require surgical staging. Clinical history, bilaterality, and marked nuclear atypia with numerous mitotic figures should raise suspicion for a metastatic carcinoma. An adult-type tumor with a microfollicular pattern can mimic a metastatic or primary typical or atypical carcinoid tumor. Carcinoid tumors have round nuclei with smooth nuclear membrane and stippled chromatin, in contrast to oval nuclei and nuclear groove in GCT (Figs. 12C, 12D). In the distinction of the sarcomatoid type from cellular fibroma/fibrosarcoma, the characteristic nuclear features and identification of other morphologic pat-
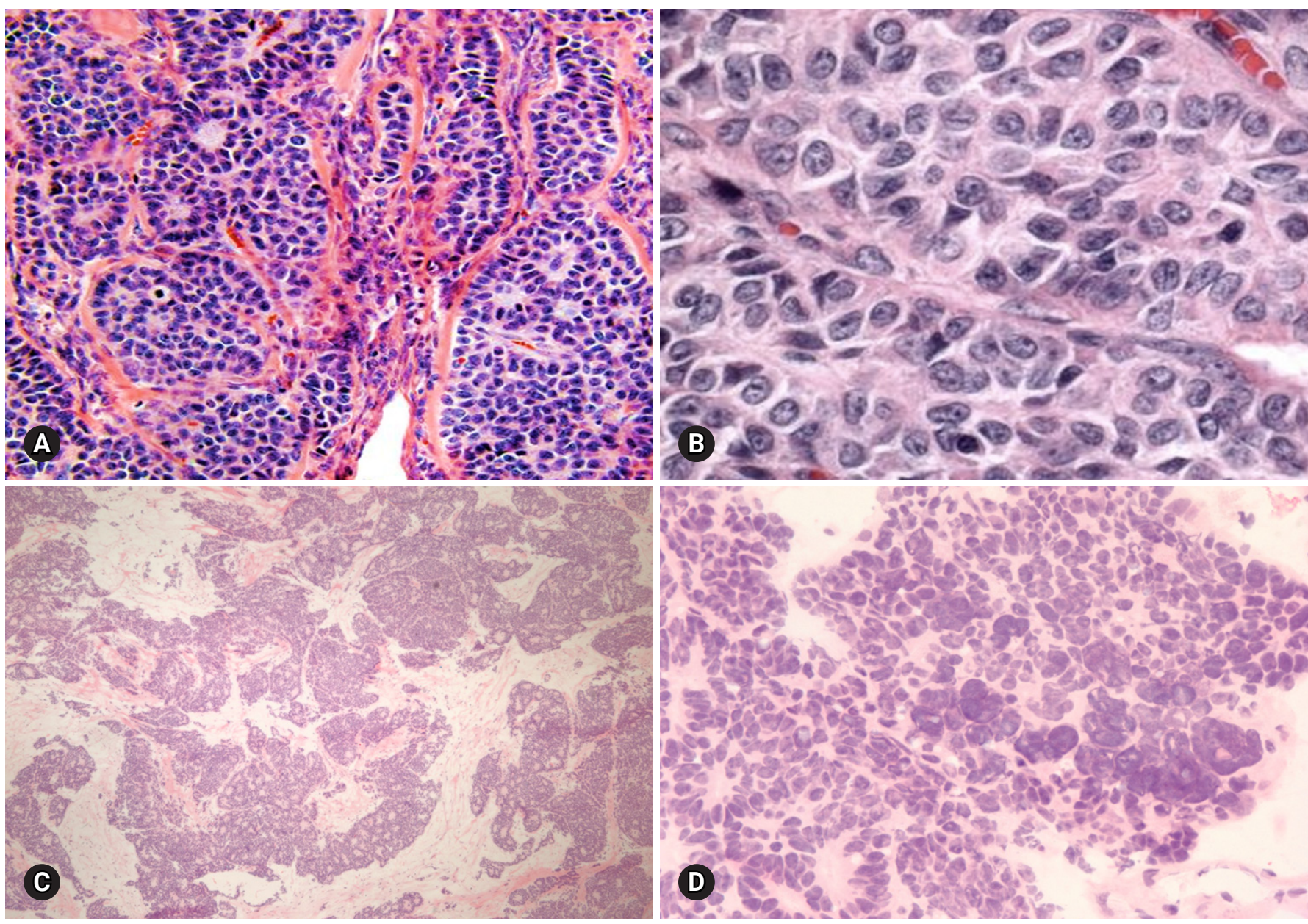

Fig. 12. Adult granulosa cell tumor typically shows microfollicular pattern with Call-Exner bodies (A), and the tumor cells are oval and have angular nucleus with groove (B). Metastatic atypical carcinoid may show microfollicular structure, but the nuclei of the tumor cells are different from those of granulosa cell tumor. 
terns of GCT can be helpful. If the frozen diagnosis is adult GCT, evaluation of the endometrium is also recommended.

One SLCT and 1 metastatic atypical carcinoid were diagnosed as adult GCT on FS.

\section{Sertoli-Leydig cell tumors}

SLCT is a rare tumor that commonly occurs in young patients and presents androgenic symptoms. Most of the tumors are unilateral, solid, and tan-yellow, or partly cystic in tumors with a retiform pattern or a mucinous heterologous component. Microscopically, the tumor is characterized by open or solid tubules, cords and diffuse sheets of primitive stromal appearance of Sertoli cells, and a variable degree of Leydig cells (Fig. 13A). The tumors are classified into well, moderately, and poorly differentiated groups, according to the differentiation of Sertoli cells. Approximately $20 \%$ of the tumors have heterologous components such as mucinous epithelium, cartilage, or skeletal muscle, and approximately $15 \%$ of SLCTs show a retiform pattern [4,27]. The prognosis of patients depends on the differentiation and on the presence of retiform and heterologous components [28]. Conservative fertility-sparing surgery in young patients with stage 1 tumor includes unilateral SO and staging procedure; however, bilateral SO, total hysterectomy, and complete surgical staging are performed in patients who do not wish to preserve fertility [22].

The differential diagnosis between moderately and poorly differentiated SLCT and adult GCT is not easy on FS, but the surgical management is basically similar (Fig. 13B). In distinguishing from ovarian primary sertoliform EC, the presence of unilaterali- ty, younger age, absence of squamous differentiation, and presence of Leydig cells can be helpful in the diagnosis of SLCT. Surgical staging is more extensive in ovarian epithelial malignancy. Among 2 SLCTs, 1 tumor was diagnosed as adult GCT on FS.

\section{Germ cell tumors}

Germ cell tumors comprise the second most common type of tumors; however, the majority of tumors are benign mature cystic teratomas. Malignant germ cell tumors are relatively rare, but the recognition of these tumors and their distinction from aggressive epithelial carcinomas are crucial. Because most malignant germ cell tumors occur in young patients, fertility-sparing surgery is important. Fortunately, adjuvant chemotherapy is now the standard management for these tumors. The patient's age, hormonal manifestations such as precocious puberty, and serum tumor markers can provide the diagnostic clues for the FS diagnosis. Mixed germ cell tumors of 2 or more types of malignant germ cell tumors may occur. Identification of different histological components is important for the treatment and prognosis. However, it is not crucial on FS. "Malignant germ cell tumor, probably mixed components" is sufficient for the FS diagnosis.

\section{Mature and immature teratoma}

Teratoma is a very common ovarian tumor. Mature teratomas account for $>95 \%$ of all teratoma and most commonly occur during the reproductive ages. On the other hand, immature teratomas are relatively rare, representing $1-3 \%$ of all teratomas, and most commonly occur during the first 2 decades of life. Bilateral-
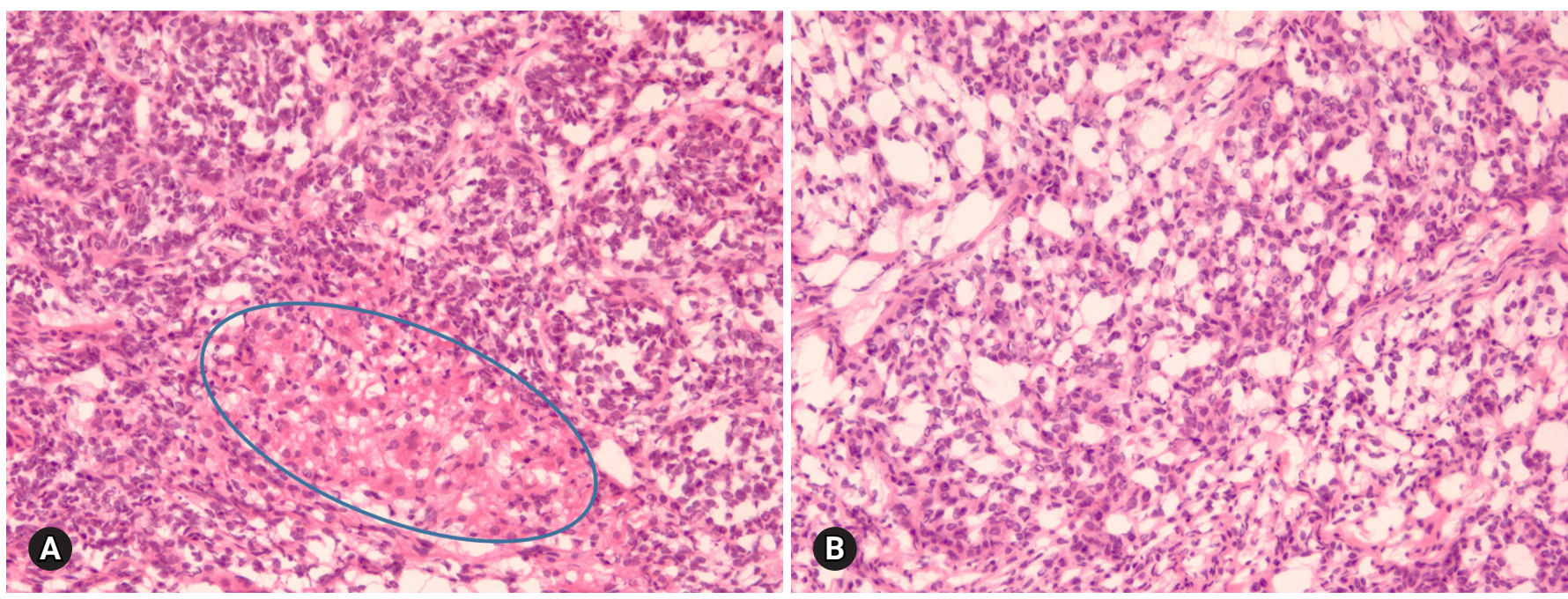

Fig. 13. Sertoli-Leydig cell tumor (A) has sex cord component similar to adult granulosa cell tumor (B), but Leydig cell component is present in Sertoli-Leydig cell tumor (circle in A). 
ity is observed in $10-15 \%$ of the cases. Mature teratoma is almost always cystic, but rarely solid. Immature teratoma is solid and cystic. Microscopically, the neural elements in mature teratoma may mimic the primitive immature neuroepithelial tissue of immature teratoma [29]. Immature mesenchymal and endodermal elements are sometimes difficult to detect on FS. The diagnosis of immature teratoma should rely on the identification of immature neuroepithelial tissue (Fig. 14A). Carcinosarcoma, composed of carcinomatous and sarcomatous components, may be considered in the differential diagnosis, but it most often occurs in the postmenopausal ages (Fig. 14B). Monodermal teratomas include struma ovarii and carcinoid tumor. Malignant transformation of mature teratoma rarely occurs, most often in postmenopausal patients; however, when it occurs, full surgical staging is required [30]. The FS diagnosis of mature cystic teratoma is straightforward, but adequate sampling from solid areas including the Rokitansky protuberance should be done.

\section{Dysgerminoma}

Dysgerminoma is the most common malignant germ cell tumor, occurring most often during the $2^{\text {nd }}$ and $3^{\text {rd }}$ decades of life. It is a rapidly growing tumor and may arise in dysgenetic gonads with gonadoblastoma. The tumor is unilateral in $80-90 \%$ of the cases, and is large, solid, and flesh and tan-yellow in color (Fig. 15A). Microscopically, the tumor is characterized by solid nests, sheets, or cords of relatively uniform polygonal cells with abundant clear or eosinophilic cytoplasm and prominent nucleoli, with intersecting fibrous septae infiltrated with lymphocytes (Fig. 15B).

On FS, contralateral ovarian biopsy should be performed to rule out bilateral tumor or underlying dysgenetic gonads and/or gonadoblastoma, for which bilateral oophorectomy is indicated. However, fertility-sparing surgery (unilateral SO with peritoneal biopsy and lymph node sampling) is adequate for unilateral dysgerminoma [31]. The distinction from other malignant germ cell tumors, yolk sac tumor and embryonal carcinoma is not crucial on FS. The surgical management is essentially identical. The most important issue concerning dysgerminoma on FS is its differentiation from diffuse large cell lymphoma, because the management of lymphoma is basically nonsurgical (Figs. 15C, 15D).

One of 2 dysgerminomas was diagnosed as metastatic carcinoma, whereas 1 malignant lymphoma was interpreted as dysgerminoma on FS in this review.

\section{Yolk sac tumor}

Yolk sac tumor accounts for $20 \%$ of malignant germ cell tumors and occurs during the $2^{\text {nd }}$ and $3^{\text {rd }}$ decades of life. This tumor may be a component of a mixed germ cell tumor and rarely occurs in association with a surface epithelial tumor, usually endometrioid $[32,33]$. The serum alpha-fetoprotein level is elevated. The tumor is nearly always unilateral, large, solid, and tan-yellow, with areas of necrosis, hemorrhage, and cyst formation. Microscopically, various growth patterns, such as reticular pattern, endodermal sinus pattern with Schiller-Duval bodies, solid pattern, alveolar glandular pattern, and other rare polyvesicular vitelline or hepatoid patterns, are present within the same tumor (Fig. 16).

The distinction from other malignant germ cell tumors is not crucial on FS, because the surgical management is similar. The most important entity in the differential diagnosis is clear cell
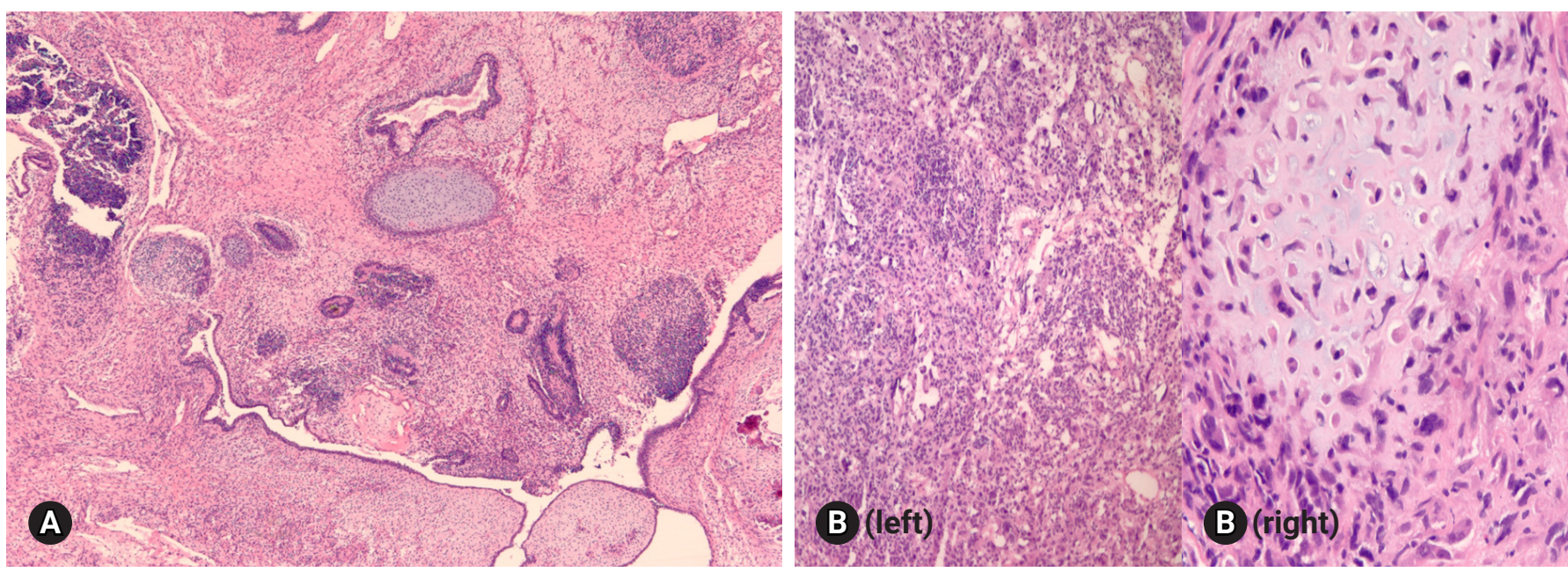

Fig. 14. Immature teratoma shows primitive neuroepithelial component, in addition to immature epithelial and mesenchymal tissues including immature cartilage $(A)$, whereas carcinosarcoma is composed of carcinomatous ( $B$, left) and sarcomatous components with malignant-looking cartilage ( $\mathrm{B}$, right). 

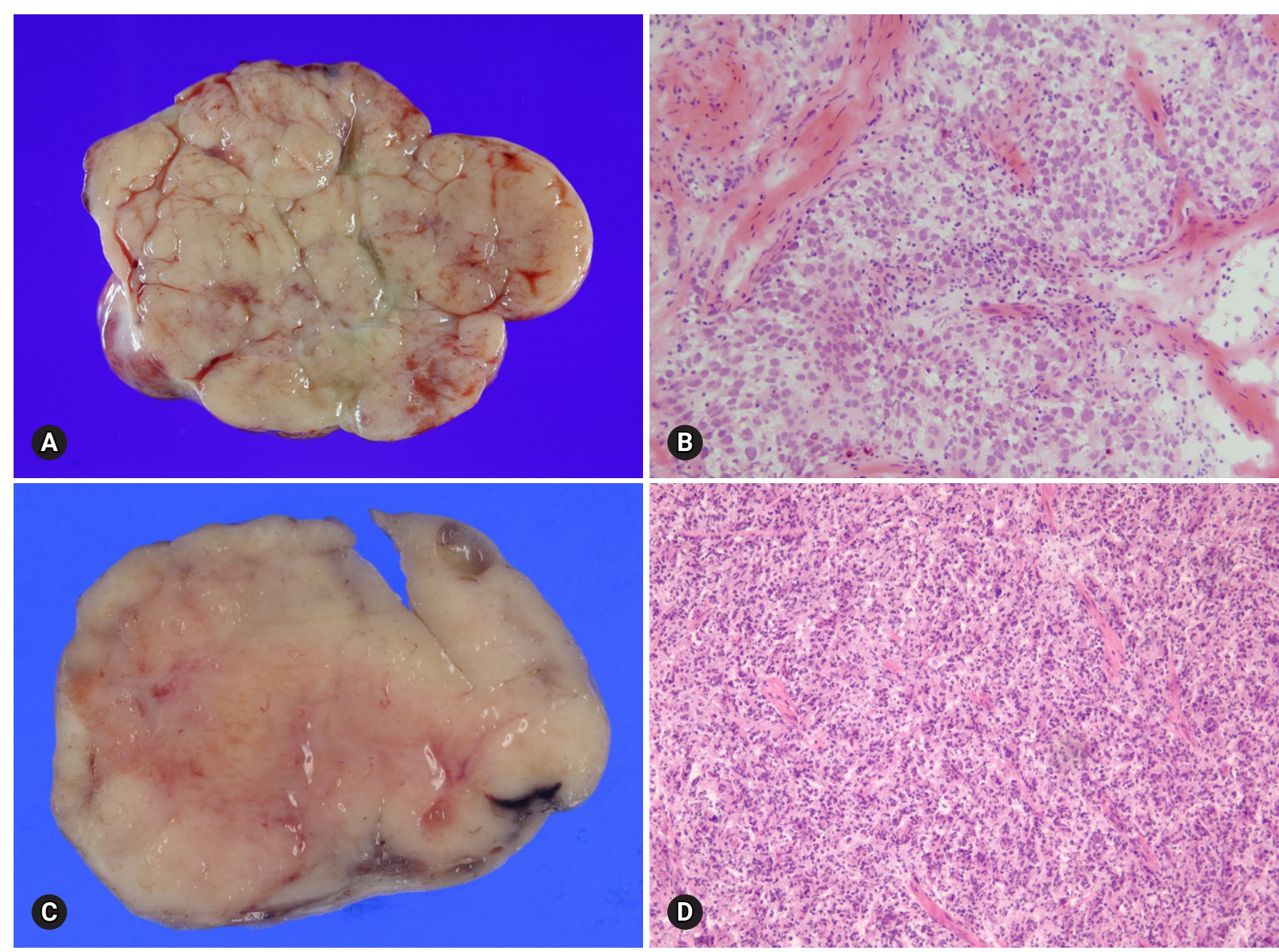

Fig. 15. Dysgerminoma shows pale gray, solid, and lobulated cut surface (A), and histologically is characterized by sheets of large polygonal cells separated by fibrous septa with infiltration of lymphocytes on frozen section (B). Malignant lymphoma shows homogeneous, fish flesh cut surface (C), and diffuse infiltration of large lymphoid cells (D).
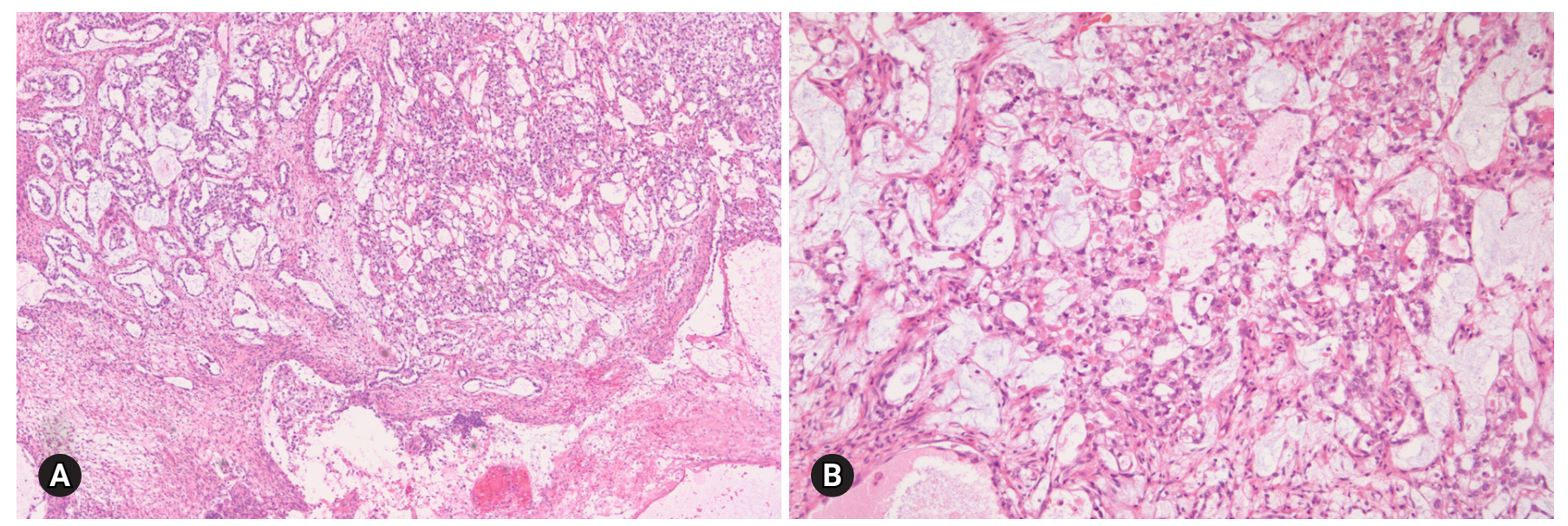

Fig. 16. Yolk sac tumor shows reticular, alveolar and papillary growth patterns (A). The tumor cells are large, pleomorphic and contain hyaline globules (B). 
carcinoma, which requires more extensive staging. Clear cell carcinoma usually occurs in older ages and is commonly associated with endometriosis.

\section{Choriocarcinoma}

Choriocarcinoma is a rare type of malignant germ cell tumor. Nongestational choriocarcinoma occurs in children and young adults and may be mixed with other malignant germ cell tumor components, whereas gestational choriocarcinoma occurs in older age groups. Elevated serum beta-human chorionic gonadotropin level and isosexual pseudoprecocity may be present. The tumor is typically large and solid and cystic, often with hemorrhage and necrosis. Microscopically, a biphasic pattern of cytotrophoblasts and multinucleated syncytiotrophoblasts is typically present (Fig. 17). Other malignant germ cell tumors may show isolated syncytiotrophoblastic giant cells, but the distinction on FS is not crucial. However, poorly differentiated surface epithelial carcinoma with trophoblastic differentiation, typically occurring in postmenopausal patients, should be differentiated because it requires more aggressive surgery [34]. One choriocarcinoma in this review was interpreted as torsion because of the presence of extensive hemorrhagic necrosis.

\section{Metastatic tumors}

Metastatic tumors account for about $15 \%$ of ovarian malignancies and 6-7\% of all adnexal masses [35]. Among 467 cases of ovarian tumors, 21 (4.5\%) cases were metastatic tumors. Ovarian metastasis most commonly originates from the gastrointestinal tract (colon, appendix, stomach, and pancreatobiliary tract).
The breast is the most frequent primary site among the nongastrointestinal origins [36]. Endometrial and cervical carcinoma may metastasize to the ovary. Metastatic ovarian tumor can be present synchronously or metachronously with the primary neoplasm, but an ovarian tumor may represent the first manifestation of an occult nonovarian primary neoplasm. Metastatic tumors are bilateral in approximately $70 \%$ of the cases, are small in size $(<10 \mathrm{~cm})$, and show superficial surface involvement and multinodular growth; however, they can be unilateral, large, and cystic, resembling a primary ovarian neoplasm [37]. Microscopically, metastatic tumors are characterized by surface implants, multinodularity, lymphovascular invasion, and extensive extraovarian spread. However, the microscopic appearance of the metastasis is variable depending on the primary tumor, although the histologic features of metastatic tumors are not always identical to those of the primary tumors.

Among 19 metastatic tumors excluding 2 malignant lymphomas, 17 tumors were adenocarcinomas, 1 tumor was a squamous cell carcinoma, and 1 was an atypical carcinoid. The primary sites were the stomach in 8 cases, the colon in 4 cases, the uterine cervix in 3 cases, and unknown in 3 cases. The 3 tumors among 19 metastatic tumors were misinterpreted as 1 mucinous carcinoma, $1 \mathrm{EC}$, and $1 \mathrm{GCT}$.

\section{Colorectal adenocarcinoma}

Metastatic adenocarcinoma from the colorectum is often bilateral and solid or solid and cystic with a tan-yellow or gray cut surface (Fig. 18A). Ovarian surface involvement and multinodular growth pattern are commonly seen. Histologically, the tumor shows variable-sized, often cystic, irregular glands. A cribriform
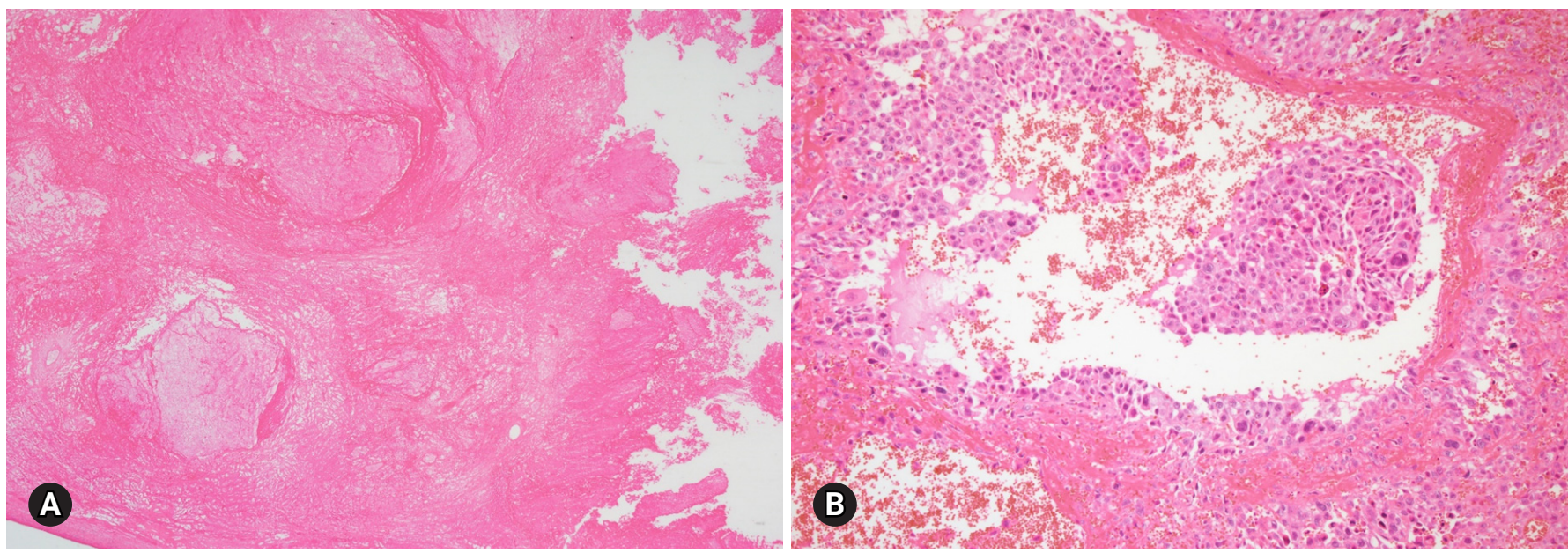

Fig. 17. Choriocarcinoma shows extensive hemorrhagic necrosis (A). Viable tumor nest is composed of two cell pattern of cytotrophoblasts and syncytiotrophoblasts (B). 
architecture with dirty necrosis is called a garland pattern. The differential diagnosis from primary ovarian mucinous and endometrioid adenocarcinoma is important (Fig. 18B). Bilaterality, size $<10 \mathrm{~cm}$, ovarian surface involvement, and a multinodular growth pattern suggest metastasis rather than a primary ovarian tumor [37]. If the tumor shows mucinous differentiation, intraoperative surgical evaluation of the abdominal cavity is recommended to find the possible primary lesion of the metastasis. Squamous differentiation in adenocarcinoma indicates primary ovarian EC rather than metastasis from a primary colorectal tumor.

\section{Signet ring cell carcinoma}

The primary site of metastatic signet ring cell carcinoma is most commonly the stomach (70\%), but less commonly the colorec- tum, appendix, breast, and pancreaticobiliary system [38,39]. The tumor is bilateral, grossly solid, and tan-yellow or white, but may also be firm or soft, and gelatinous (Fig. 19A). Histologically, the tumor shows a pseudo-lobular pattern with hypercellular and hypocellular areas or diffusely cellular dense stroma (Fig. 19B). Signet ring cells individually infiltrate or form small clusters or tubules. Extracellular mucin may be present. On FS, signet ring cells may be missed and misinterpreted as fibroma or fibrothecoma. Primary ovarian sex cord-stromal tumors are usually unilateral.

\section{Appendiceal tumors}

The most common appendiceal tumor with ovarian involvement is low-grade mucinous neoplasm. Less commonly, intestinal-type
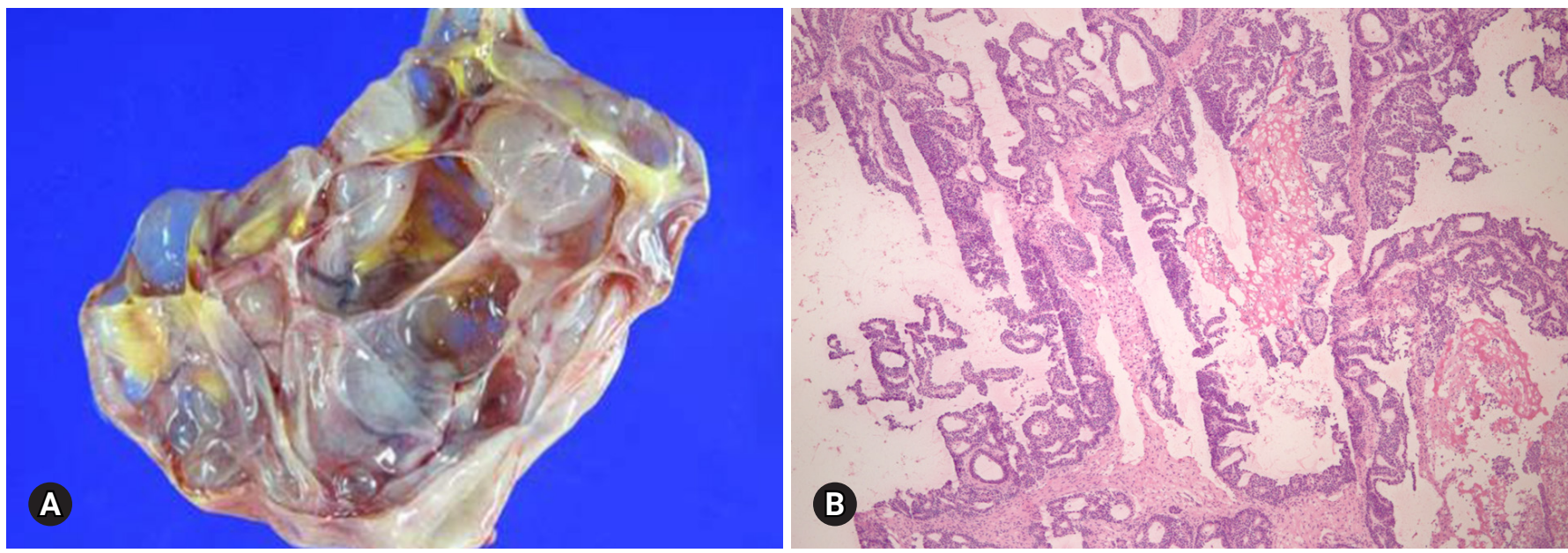

Fig. 18. Multilocular cystic metastasis from colon cancer with multifocal yellow necrotic foci (A). Garland pattern with central necrosis simulates endometrioid carcinoma on frozen section (B).
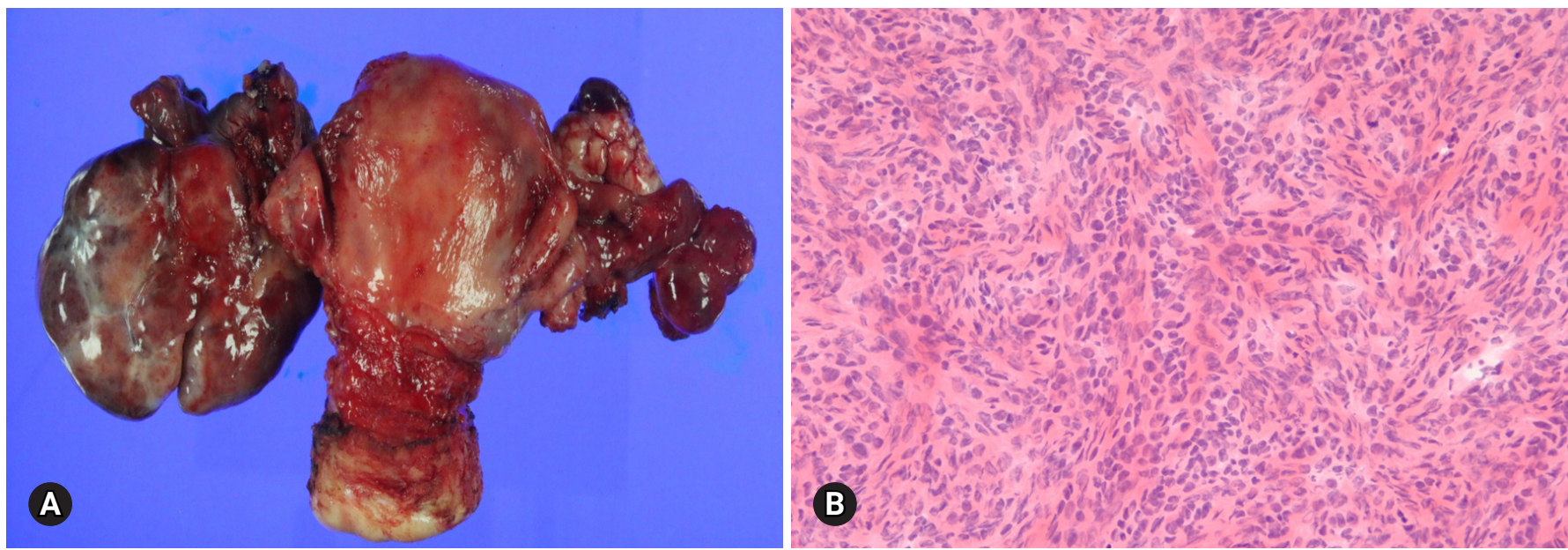

Fig. 19. Metastatic signet ring cell carcinoma involving both ovaries (A). Fibrous stroma is infiltrated with nests and cords of small tumor cells on frozen section (B). 

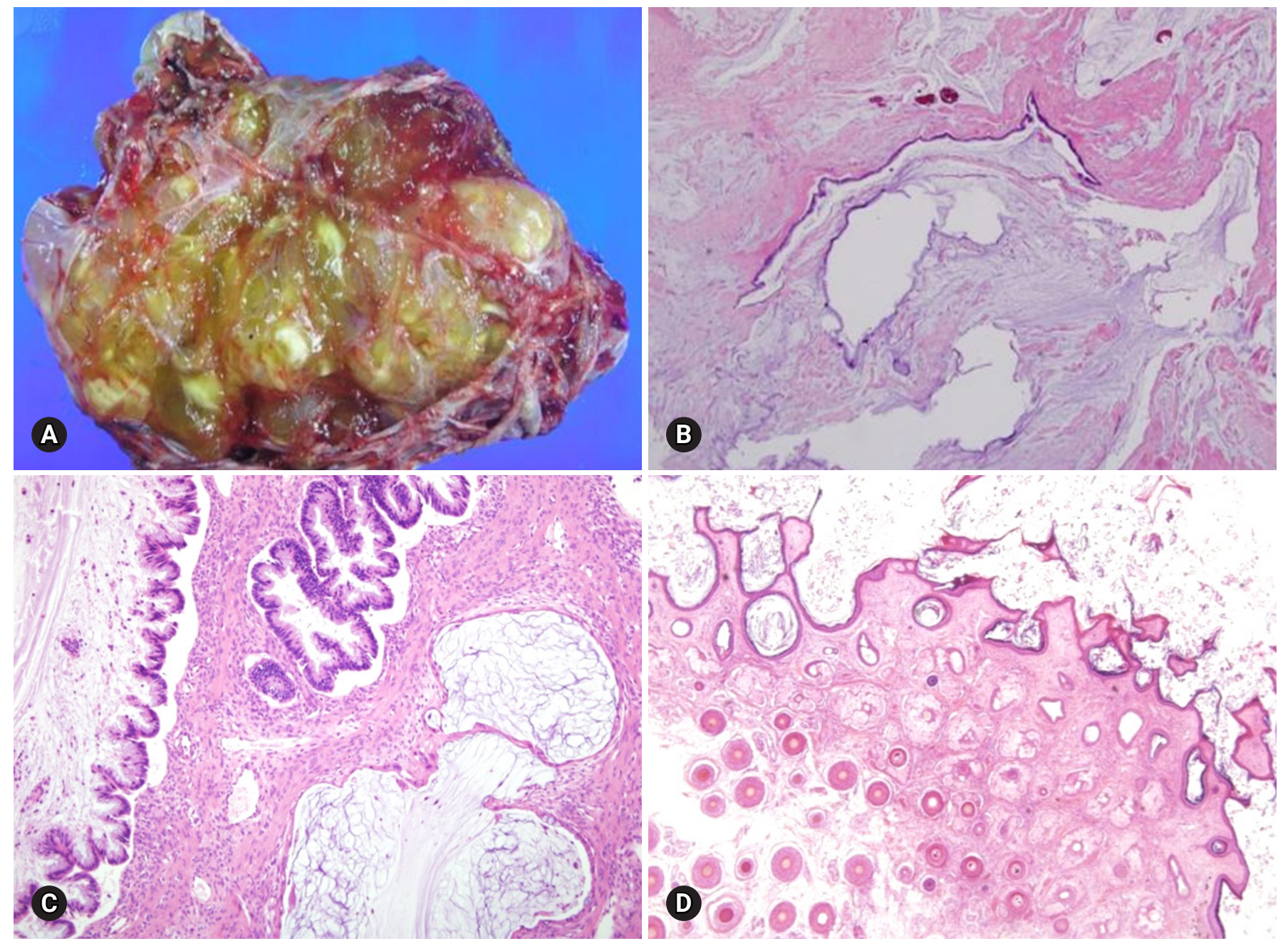

Fig. 20. Mucinous borderline tumor showing multilocular cysts filled with yellow, gelatinous material (A). Histologically, mucinous tumor represents benign (B) and borderline (C) areas and extensive pseudomyxoma ovarii. The association with mature teratoma indicates ovarian origin than appendiceal metastasis (D).

adenocarcinoma, signet ring cell carcinoma, and goblet cell carcinoid tumor may involve the ovary. Low-grade mucinous neoplasm is often associated with pseudomyxoma peritonei and is frequently bilateral, but shows predominantly right-sided involvement among unilateral cases [40]. The cut surface reveals a multilocular cyst with tan-gray, mucoid appearance and surface involvement. The appendix is dilated with mucus. Histologically, the cysts are lined by flat or undulating mucinous epithelium with spillage of mucin into the stroma (pseudomyxoma ovarii). Goblet cells may be present. The mitotic activity is not significant. The presence of pseudomyxoma peritonei, bilaterality, and ovarian surface involvement indicate appendiceal metastasis rather than primary ovarian tumor [41]. However, similar morphologic features may be present in a primary mucinous tumor associated with a mature teratoma (Fig. 20). Appendectomy and
FS evaluation of the appendix should be done even in the absence of gross abnormalities.

\section{Uterine cervical and endometrial carcinomas}

Uterine cervical adenocarcinoma and, less commonly, squamous cell carcinoma may spread to the ovary, even in cases in which the primary tumor is small and clinically unsuspected for ovarian metastasis. Histologically, the tumors may mimic primary borderline or malignant mucinous and endometrioid tumors [42]. Endometrial and ovarian involvement by endometrioid or serous carcinoma may represent a synchronous independent primary tumor or ovarian metastasis from a primary endometrial tumor. The distinction between these possibilities is impossible at the time of FS and is usually not crucial for the intraoperative surgical management. 


\section{Conclusion}

The discrepancies between frozen diagnosis and permanent diagnosis are mainly due to sampling errors and misinterpretation by pathologists but may also be due to suboptimal quality of the FS. If the diagnosis is doubtful, the gross specimen should be reexamined and additional sections should be taken, a second opinion from an experienced pathologist should be obtained, and promptly discuss with the surgeon to obtain more history information and to discuss about a difficult diagnostic interpretation.

\section{Conflicts of interest}

No potential conflicts of interest relevant to this article was reported.

\section{Acknowledgements}

I would express many thanks to Dr. Cho, Hwa Jin (Inje University Busan Paik Hospital), Dr. Lee, Jeong Hyeon (Korea University Anam Hospital), Dr. Kim, Hye Sun (Dankuk University Cheil Hospital), Dr. Lee, Jeong Hee (Gyeongsang National University Hospital) for collecting the cases.

\section{ORCID}

Insun Kim, https://orcid.org/0000-0002-1924-7893

\section{References}

1. Gal AA, Cagle PT. The 100-year anniversary of the description of the frozen section procedure. JAMA 2005;294:3135-7.

2. Krishnamurthy S, Meric-Bernstam F, Lucci A, Hwang RF, Kuerer HM, Babiera G, et al. A prospective study comparing touch imprint cytology, frozen section analysis, and rapid cytokeratin immunostain for intraoperative evaluation of axillary sentinel lymph nodes in breast cancer. Cancer 2009;115:1555-62.

3. Novis DA, Zarbo RJ. Interinstitutional comparison of frozen section turnaround time. A College of American Pathologists Q-Probes study of 32868 frozen sections in 700 hospitals. Arch Pathol Lab Med 1997;121:559-67.

4. Kurman RJ; International Agency for Research on Cancer (IARC). WHO classification of tumours of female reproductive organs. 4th ed. Lyon: International Agency for Research on Cancer; 2014.

5. Bige O, Demir A, Saygili U, Gode F, Uslu T, Koyuncuoglu M.
Frozen section diagnoses of 578 ovarian tumors made by pathologists with and without expertise on gynecologic pathology. Gynecol Oncol 2011;123:43-6.

6. Malipatil R, Crasta JA. How accurate is intraoperative frozen section in the diagnosis of ovarian tumors? J Obstet Gynaecol Res 2013;39:710-3.

7. Rodríguez IM, Prat J. Mucinous tumors of the ovary: a clinicopathologic analysis of 75 borderline tumors (of intestinal type) and carcinomas. Am J Surg Pathol 2002;26:139-52.

8. Lee KR, Young RH. The distinction between primary and metastatic mucinous carcinomas of the ovary: gross and histologic findings in 50 cases. Am J Surg Pathol 2003;27:281-92.

9. Bagué S, Rodríguez IM, Prat J. Sarcoma-like mural nodules in mucinous cystic tumors of the ovary revisited: a clinicopathologic analysis of 10 additional cases. Am J Surg Pathol 2002;26:1467-76.

10. Provenza C, Young RH, Prat J. Anaplastic carcinoma in mucinous ovarian tumors: a clinicopathologic study of 34 cases emphasizing the crucial impact of stage on prognosis, their histologic spectrum, and overlap with sarcomalike mural nodules. Am J Surg Pathol 2008;32:383-9.

11. Seidman JD, Soslow RA, Vang R, Berman JJ, Stoler MH, Sherman $\mathrm{ME}$, et al. Borderline ovarian tumors: diverse contemporary viewpoints on terminology and diagnostic criteria with illustrative images. Hum Pathol 2004;35:918-33.

12. McKenney JK, Balzer BL, Longacre TA. Patterns of stromal invasion in ovarian serous tumors of low malignant potential (borderline tumors): a reevaluation of the concept of stromal microinvasion. Am J Surg Pathol 2006;30:1209-21.

13. Bell DA, Weinstock MA, Scully RE. Peritoneal implants of ovarian serous borderline tumors. Histologic features and prognosis. Cancer 1988;62:2212-22.

14. Malpica A, Deavers MT, Lu K, Bodurka DC, Atkinson EN, Gershenson DM, et al. Grading ovarian serous carcinoma using a two-tier system. Am J Surg Pathol 2004;28:496-504.

15. Roth LM, Emerson RE, Ulbright TM. Ovarian endometrioid tumors of low malignant potential: a clinicopathologic study of 30 cases with comparison to well-differentiated endometrioid adenocarcinoma. Am J Surg Pathol 2003;27:1253-9.

16. Bell KA, Kurman RJ. A clinicopathologic analysis of atypical proliferative (borderline) tumors and well-differentiated endometrioid adenocarcinomas of the ovary. Am J Surg Pathol 2000;24:1465-79.

17. Brescia RJ, Dubin N, Demopoulos RI. Endometrioid and clear cell carcinoma of the ovary. Factors affecting survival. Int J Gynecol Pathol 1989;8:132-8.

18. Zaino RJ, Unger ER, Whitney C. Synchronous carcinomas of the uterine corpus and ovary. Gynecol Oncol 1984;19:329-35. 
19. Roth LM, Langley FA, Fox H, Wheeler JE, Czernobilsky B. Ovarian clear cell adenofibromatous tumors. Benign, of low malignant potential, and associated with invasive clear cell carcinoma. Cancer 1984;53:1156-63.

20. Scarfone G, Bergamini A, Noli S, Villa A, Cipriani S, Taccagni G, et al. Characteristics of clear cell ovarian cancer arising from endometriosis: a two center cohort study. Gynecol Oncol 2014;133:480-4.

21. Irving JA, Alkushi A, Young RH, Clement PB. Cellular fibromas of the ovary: a study of 75 cases including 40 mitotically active tumors emphasizing their distinction from fibrosarcoma. Am J Surg Pathol 2006;30:929-38.

22. Bhat RA, Lim YK, Chia YN, Yam KL. Sertoli-Leydig cell tumor of the ovary: analysis of a single institution database. J Obstet Gynaecol Res 2013;39:305-10.

23. van Meurs HS, Bleeker MC, van der Velden J, Overbeek LI, Kenter GG, Buist MR. The incidence of endometrial hyperplasia and cancer in 1031 patients with a granulosa cell tumor of the ovary: long-term follow-up in a population-based cohort study. Int J Gynecol Cancer 2013;23:1417-22.

24. Young RH, Dickersin GR, Scully RE. Juvenile granulosa cell tumor of the ovary. A clinicopathological analysis of 125 cases. Am J Surg Pathol 1984;8:575-96.

25. Schumer ST, Cannistra SA. Granulosa cell tumor of the ovary. J Clin Oncol 2003;21:1180-9.

26. Iavazzo C, Gkegkes ID, Vrachnis N. Fertility sparing management and pregnancy in patients with granulosa cell tumour of the ovaries. J Obstet Gynaecol 2015;35:331-5.

27. Young RH, Scully RE. Ovarian Sertoli-Leydig cell tumors. A clinicopathological analysis of 207 cases. Am J Surg Pathol 1985;9:543-69.

28. Gui T, Cao D, Shen K, Yang J, Zhang Y, Yu Q, et al. A clinicopathological analysis of 40 cases of ovarian Sertoli-Leydig cell tumors. Gynecol Oncol 2012;127:384-9.

29. Norris HJ, Zirkin HJ, Benson WL. Immature (malignant) teratoma of the ovary: a clinical and pathologic study of 58 cases. Cancer 1976;37:2359-72.

30. Stamp GW, McConnell EM. Malignancy arising in cystic ovarian teratomas. A report of 24 cases. Br J Obstet Gynaecol 1983;90:671-5.

31. Eskander RN, Randall LM, Berman ML, Tewari KS, Disaia PJ, Bristow RE. Fertility preserving options in patients with gynecologic malignancies. Am J Obstet Gynecol 2011;205:103-10.
32. Nogales FF, Preda O, Nicolae A. Yolk sac tumours revisited. A review of their many faces and names. Histopathology 2012;60:1023-33.

33. Roth LM, Talerman A, Levy T, Sukmanov O, Czernobilsky B. Ovarian yolk sac tumors in older women arising from epithelial ovarian tumors or with no detectable epithelial component. Int J Gynecol Pathol 2011;30:442-51.

34. Oliva E, Andrada E, Pezzica E, Prat J. Ovarian carcinomas with choriocarcinomatous differentiation. Cancer 1993;72:2441-6.

35. Ulbright TM, Roth LM, Stehman FB. Secondary ovarian neoplasia. A clinicopathologic study of 35 cases. Cancer 1984;53:1164-74.

36. Mazur MT, Hsueh S, Gersell DJ. Metastases to the female genital tract. Analysis of 325 cases. Cancer 1984;53:1978-84.

37. Yemelyanova AV, Vang R, Judson K, Wu LS, Ronnett BM. Distinction of primary and metastatic mucinous tumors involving the ovary: analysis of size and laterality data by primary site with reevaluation of an algorithm for tumor classification. Am J Surg Pathol 2008;32:128-38.

38. Young RH. From krukenberg to today: the ever present problems posed by metastatic tumors in the ovary: part I. Historical perspective, general principles, mucinous tumors including the krukenberg tumor. Adv Anat Pathol 2006;13:205-27.

39. Kiyokawa T, Young RH, Scully RE. Krukenberg tumors of the ovary: a clinicopathologic analysis of 120 cases with emphasis on their variable pathologic manifestations. Am J Surg Pathol 2006;30:277-99.

40. Young RH, Gilks CB, Scully RE. Mucinous tumors of the appendix associated with mucinous tumors of the ovary and pseudomyxoma peritonei. A clinicopathological analysis of 22 cases supporting an origin in the appendix. Am J Surg Pathol 1991;15:415-29.

41. Stewart CJ, Ardakani NM, Doherty DA, Young RH. An evaluation of the morphologic features of low-grade mucinous neoplasms of the appendix metastatic in the ovary, and comparison with primary ovarian mucinous tumors. Int J Gynecol Pathol 2014;33:1-10.

42. Ronnett BM, Yemelyanova AV, Vang R, Gillks CB, Miller D, Gravitt PE, et al. Endocervical adenocarcinomas with ovarian metastases: analysis of 29 cases with emphasis on minimally invasive cervical tumors and the ability of the metastases to simulate primary ovarian neoplasms. Am J Surg Pathol 2008;32:1835-53. 